\title{
Oriented Interval Greedoids
}

\author{
Franco Saliola $\cdot$ Hugh Thomas
}

Received: 6 October 2009 / Revised: 9 September 2011 / Accepted: 13 September 2011 /

Published online: 8 November 2011

(C) Springer Science+Business Media, LLC 2011

\begin{abstract}
We propose a definition of an oriented interval greedoid that simultaneously generalizes the notion of an oriented matroid and the construction on antimatroids introduced by L.J. Billera, S.K. Hsiao, and J.S. Provan in Enumeration in convex geometries and associated polytopal subdivisions of spheres (Discrete Comput. Geom. 39(1-3):123-137, 2008). As for oriented matroids, associated to each oriented interval greedoid is a spherical simplicial complex whose face enumeration depends only on the underlying interval greedoid.
\end{abstract}

Keywords Interval greedoid · Oriented matriod · Antimatroid · Convex geometry · Sphericity theorem $\cdot \mathrm{CW}$ sphere

\section{Introduction}

Consider a hyperplane arrangement in $\mathbb{R}^{n}$, with all of the hyperplanes containing the origin. Intersecting this arrangement with a sphere centered at the origin, one obtains a regular cell decomposition of the sphere. Taking the barycentric subdivision, one obtains a spherical simplicial complex.

Oriented matroids are a generalization of real hyperplane arrangements; the Sphericity Theorem of Folkman and Lawrence [21] for oriented matroids says that any oriented matroid induces a certain regular cell decomposition of the sphere (and thus also a spherical simplicial complex) just as hyperplane arrangements do. (Terms not defined in the introduction will be defined later in the paper.)

\section{F. Saliola ( $\varangle)$}

Département de Mathématiques, Université du Québec à Montréal, Montréal, QC, H3C 3P8, Canada

e-mail: saliola.franco@uqam.ca

H. Thomas

Department of Mathematics and Statistics, University of New Brunswick, Fredericton, NB,

E3B 5A3, Canada

e-mail: hugh@math.unb.ca 
Billera, Hsiao, and Provan showed in [4] that there is also a certain spherical simplicial complex associated to a convex geometry (or antimatroid). These simplicial complexes are not a special case of the spheres arising from oriented matroids, but they are similar in some respects (see Sect. 6.3 in particular).

The goal of this paper is to provide a general theory which includes both of these as special cases. Following a suggestion in [4] (attributed to Anders Björner), our approach is via the notion of interval greedoid. The precise definition appears in the next section, but for now, it suffices to know that interval greedoids are a common generalization of matroids and antimatroids.

In this paper, we define the notion of an oriented interval greedoid. This is an additional structure on top of the interval greedoid structure. For a given interval greedoid, there may be no such additional structure possible, or one, or more than one.

For an interval greedoid which is a matroid, an oriented interval greedoid structure amounts to (the collection of covectors defining) an oriented matroid. In contrast, if the underlying interval greedoid is an antimatroid, it always admits exactly one oriented interval greedoid structure.

Our main result is an analogue of the Sphericity Theorem for oriented interval greedoids, providing a CW-sphere and (by barycentric subdivision) a spherical simplicial complex associated to any oriented interval greedoid. Our proof is based on the proof of the Sphericity Theorem given in [13]. The spherical simplicial complex associated to the unique oriented structure for an antimatroid, coincides with that constructed by [4].

Along the way, we give versions for oriented interval greedoids of a number of constructions for oriented matroids, such as restriction and contraction.

\section{Interval Greedoids}

In this section we recall basic notions and results on interval greedoids. Much of the material in this section, except for Sect. 2.2.5, is drawn from [19] or [22]. The maps $\mu$ and $\xi$ introduced in Sect. 2.2.5 seem to be new.

\subsection{Definition}

Let $E$ denote a finite set and $\mathcal{F}$ a set of subsets of $E$. An interval greedoid is a pair $(E, \mathcal{F})$ satisfying the following properties for all $X, Y, Z \in \mathcal{F}$ :

(IG1) If $X \neq \varnothing$, then there exists an $x \in X$ such that $X-x \in \mathcal{F}$.

(IG2) If $|X|>|Y|$, then there exists an $x \in X \backslash Y$ such that $Y \cup x \in \mathcal{F}$.

(IG3) If $X \subseteq Y \subseteq Z$ and $e \in E \backslash Z$ with $X \cup e \in \mathcal{F}$ and $Z \cup e \in \mathcal{F}$, then $Y \cup e \in \mathcal{F}$.

The set $E$ is called the ground set of the interval greedoid $(E, \mathcal{F})$. Elements of $\mathcal{F}$ are called the feasible sets of $(E, \mathcal{F})$. If $\mathcal{F}$ is a non-empty collection of subsets of $E$ satisfying (IG1), then $\mathcal{F}$ is said to be an accessible set system. A greedoid is a pair $(E, \mathcal{F})$ that satisfies (IG1) and (IG2). In the literature, (IG3) is often called the interval property.

We review here several important classes of interval greedoids. 


\subsubsection{Lower Interval Greedoids (Matroids)}

A greedoid $(E, \mathcal{F})$ satisfying the following strengthening of (IG3) is called a lower interval greedoid.

(LIP) Suppose $X, Y \in \mathcal{F}$ with $X \subseteq Y$. If $e \in E \backslash Y$ and $Y \cup e \in \mathcal{F}$, then $X \cup e \in \mathcal{F}$.

The reader familiar with the notion of a matroid will recognize that the notions of lower interval greedoid and matroid coincide.

This notion is an abstraction of linear independence of vectors. For example, let $V=\mathbb{R}^{2}, \vec{x}=(-3,1), \vec{y}=(2,1)$ and $\vec{z}=(4,1)$, and let $\mathcal{F}$ be the collection of subsets of $E=\{\vec{x}, \vec{y}, \vec{z}\}$ that consist of linearly independent vectors:

$$
\mathcal{F}=\{\varnothing,\{\vec{x}\},\{\vec{y}\},\{\vec{z}\},\{\vec{x}, \vec{y}\},\{\vec{x}, \vec{z}\},\{\vec{y}, \vec{z}\}\} .
$$

Then $(E, \mathcal{F})$ is a lower interval greedoid. Such lower interval greedoids are called vector matriods.

\subsubsection{Upper Interval Greedoids (Antimatroids and Convex Geometries)}

A greedoid $(E, \mathcal{F})$ satisfying the following property is called an upper interval greedoid.

(UIP) Suppose $X, Y \in \mathcal{F}$ with $X \subseteq Y$. If $e \in E \backslash Y$ and $X \cup e \in \mathcal{F}$, then $Y \cup e \in \mathcal{F}$.

Upper interval greedoids arise as complements of the closed sets of a convex geometry. A convex geometry is a pair $(E, \tau)$, where $E$ is a finite set and $\tau: 2^{E} \rightarrow 2^{E}$ is an increasing, monotone and idempotent function, satisfying:

(AE) If $x, y \notin \tau(X), x \neq y$, and $y \in \tau(X \cup x)$, then $x \notin \tau(X \cup y)$.

The subsets $A \subseteq E$ satisfying $\tau(A)=A$ are called closed sets of the convex geometry. The extreme points $\operatorname{ext}(A)$ of $A \subseteq E$ are the points $x \in A$ satisfying $x \notin \tau(A \backslash x)$. If $(E, \tau)$ is a convex geometry, then the complements of the closed sets of $E$ are the feasible sets of an upper interval greedoid on the ground set $E$. Conversely, every upper interval greedoid arises from a convex geometry; see [22, Theorem III.1.3] or [19, Proposition 8.7.3].

The canonical example is a finite subset $E \subseteq \mathbb{R}^{n}$ with $\tau(A)=\operatorname{conv}(A) \cap E$, where $\operatorname{conv}(A)$ is the convex hull of the points in $A$. As an example, let $x, y, z$ be three colinear points in the plane, with $y$ between $x$ and $z$, and consider the convex geometry with closure operator $\tau(X)=\operatorname{conv}(X) \cap\{x, y, z\}$. The closed sets are the subsets

$$
\varnothing,\{x\},\{y\},\{z\},\{x, y\},\{y, z\},\{x, y, z\} .
$$

Then $(E, \mathcal{F})$ is an upper interval greedoid, where $E=\{x, y, z\}$ and $\mathcal{F}$ is

$$
\mathcal{F}=\{\{x, y, z\},\{y, z\},\{x, z\},\{x, y\},\{z\},\{x\}, \varnothing\} .
$$

Remark 2.1 Upper interval greedoids have been studied under several different names including antimatroid, APS-structures, discs, and shelling structures. Some care is required in reading the literature, as some authors have used the term antimatroid for a convex geometry. By antimatroid, we will always mean an upper interval greedoid. 


\subsubsection{Interval Greedoids from Semimodular Lattices}

If $x$ and $y$ are two elements of a poset, then we write $x \lessdot y$ to indicate that $x<y$ and that there does not exist an element $z$ in the poset satisfying $x<z<y$.

Let $L$ be a finite lattice. $L$ is said to be (lower) semimodular if it has the following property for all $x \neq y \in L$ : if $x \lessdot z$ and $y \lessdot z$ for some $z \in L$, then $x \wedge y \lessdot x$ and $x \wedge y \lessdot y$. An element $e \in L$ such that $e \neq \hat{1}$ is called meet-irreducible if $e=x \wedge y$ implies $x=e$ or $y=e$.

Proposition 2.2 [19, Theorem 8.8.7] Suppose L is a finite lower semimodular lattice. Let $E$ be the set of meet-irreducible elements of $L$, and let

$$
\mathcal{F}=\left\{\left\{e_{1}, e_{2}, \ldots, e_{k}\right\} \subseteq E: \hat{1}>e_{1}>\left(e_{1} \wedge e_{2}\right)>\cdots>\left(e_{1} \wedge e_{2} \wedge \cdots \wedge e_{k}\right)\right\}
$$

Then $(E, \mathcal{F})$ is an interval greedoid.

\subsection{The Lattice of Flats}

\subsubsection{Feasible Orderings}

Let $(E, \mathcal{F})$ denote an interval greedoid. Let $X \in \mathcal{F}$. An ordering $x_{1}<x_{2}<\cdots<x_{r}$ of the elements of $X=\left\{x_{1}, x_{2}, \ldots, x_{r}\right\}$ is denoted by $X=\left\{x_{1}<x_{2}<\cdots<x_{r}\right\}$. Such an ordering is said to be a feasible ordering of $X$ if $\left\{x_{1}, \ldots, x_{i}\right\} \in \mathcal{F}$ for all $1 \leq i \leq r=|X|$. Repeated application of (IG1) shows that every $X \in \mathcal{F}$ has a feasible ordering.

Proposition 2.3 ([22, Chap. V, Lemma 5.1], [19, Proposition 8.2.5]) Let $(E, \mathcal{F})$ be an interval greedoid. Let $X, Y \in \mathcal{F}$ and $|X|>|Y|$. Suppose $X=\left\{x_{1}<\cdots<x_{r}\right\}$ is a feasible ordering. Then there is a subset $\left\{x_{i_{1}}<\cdots<x_{i_{k}}\right\}$ of $X \backslash Y$ of size $|X|-|Y|$ such that $Y \cup\left\{x_{i_{1}}, \ldots, x_{i_{j}}\right\} \in \mathcal{F}$ for all $1 \leq j \leq k$.

\subsubsection{Contractions}

Let $(E, \mathcal{F})$ denote an interval greedoid and let $X \in \mathcal{F}$. Let $\mathcal{F} / X$ denote the collection of subsets that can be added to $X$ preserving feasibility:

$$
\mathcal{F} / X=\{Y \subseteq E \backslash X: X \cup Y \in \mathcal{F}\} \text {. }
$$

The sets in $\mathcal{F} / X$ are the feasible sets of an interval greedoid on the ground set $\bigcup_{Y \in \mathcal{F} / X} Y$; this interval greedoid is called the contraction of $(E, \mathcal{F})$ by $X$. Properties of contractions will be further developed in later sections, but for now we record the following result, which is crucial to much of what follows.

Proposition 2.4 Suppose $(E, \mathcal{F})$ is an interval greedoid and let $A \subseteq E$. Let $U$ and $V$ be maximal with respect to inclusion among the feasible sets contained in $A$. Then $\mathcal{F} / U=\mathcal{F} / V$. 
Proof Let $U$ and $V$ be two maximal feasible sets contained in $A$. Then $|U|=|V|$ (otherwise we can enlarge the smaller one using (IG2)). Suppose $W \in \mathcal{F} / U$ with $W \neq \varnothing$. Then $U \cup W \in \mathcal{F}$. Let $U=\left\{u_{1}<\cdots<u_{r}\right\}$ be a feasible ordering of $U$. Repeated application of (IG2) to $U$ and $U \cup W$ gives a feasible ordering $\left\{u_{1}<\cdots<\right.$ $\left.u_{r}<w_{1}<\cdots<w_{s}\right\}$ of $U \cup W$. Proposition 2.3 applied to $U \cup W$ and $V$ gives an ordered subset $\left\{z_{1}<\cdots<z_{t}\right\}$ of $U \cup W$ such that $V \cup\left\{z_{1}, \ldots, z_{i}\right\} \in \mathcal{F}$ for each $1 \leq$ $i \leq t$, where $t=|U \cup W|-|V|=|W|$. If $z_{1} \in U$, then $V \cup\left\{z_{1}\right\} \in \mathcal{F}$ and $V \cup\left\{z_{1}\right\} \subseteq A$, contradicting the maximality of $V$. Therefore, $z_{1} \in W$ and the ordering of the $z_{i}$ implies $z_{i} \in W$ for all $1 \leq i \leq t$. Since $t=|W|$, we have $W=\left\{z_{1}, \ldots, z_{t}\right\}$. Thus, $V \cup W \in \mathcal{F}$, or equivalently, $W \in \mathcal{F} / V$. Reversing the roles of $U$ and $V$ gives the reverse containment $\mathcal{F} / V \subseteq \mathcal{F} / U$. Thus, $\mathcal{F} / U=\mathscr{F} / V$.

\subsubsection{Continuations}

Let $(E, \mathcal{F})$ denote an interval greedoid and let $X \in \mathcal{F}$. The set of continuations $\Gamma(X)$ of $X$ is the set of elements that can be added to $X$ preserving feasibility:

$$
\Gamma(X)=\{x \in E \backslash X: X \cup x \in \mathcal{F}\} .
$$

For vector matroids, $\Gamma(X)$ consists of those vectors from $E$ not in the span of $X$. For an antimatroid, $\Gamma(X)=\operatorname{ext}(E \backslash X)$.

If $X, Y \in \mathcal{F}$ and $\mathcal{F} / X=\mathcal{F} / Y$, then $\Gamma(X)=\Gamma(Y)$. The converse does not hold for arbitrary greedoids, but it does hold for interval greedoids.

Proposition 2.5 [22, Chap. V, Theorem 5.10] Suppose $(E, \mathcal{F})$ is an interval greedoid. Then for all $X, Y \in \mathcal{F}$, we have $\Gamma(X)=\Gamma(Y)$ if and only if $\mathcal{F} / X=\mathcal{F} / Y$.

\subsubsection{Flats}

Let $(E, \mathcal{F})$ be an interval greedoid. Define an equivalence relation on $\mathcal{F}$ by setting $X \sim Y$ if and only if $\mathcal{F} / X=\mathcal{F} / Y$. In light of Proposition 2.5, $X \sim Y$ if and only if $\Gamma(X)=\Gamma(Y)$. We write $[X]$ for the equivalence class of $X$ :

$$
[X]=\{Y \in \mathcal{F}: \mathcal{F} / X=\mathcal{F} / Y\}=\{Y \in \mathscr{F}: \Gamma(X)=\Gamma(Y)\} .
$$

These equivalence classes are called the flats of $(E, \mathcal{F})$.

The set $\Phi$ of flats of $(E, \mathcal{F})$ is a poset with partial order induced by reverse inclusion:

$$
[X] \leq[Y] \text { iff there exists } Z \in \mathcal{F} / Y \text { such that } Y \cup Z \sim X \text {. }
$$

In particular, if $Y \subseteq X$, then $[X] \leq[Y]$. (Note that some authors choose to use inclusion rather than reverse inclusion to induce the partial order on $\Phi$.)

In a vector matroid, $[X] \leq[Y]$ iff the span of $Y$ is contained in the span of $X$. The poset of flats for the convex geometry on three colinear points is illustrated in Fig. 1. For an arbitrary antimatroid, $[X]=\{X\}$ for all $X \in \mathcal{F}$, thus the poset of flats $\Phi$ for 


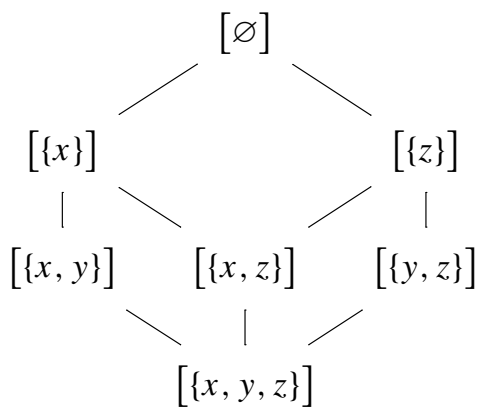

Fig. 1 The poset of flats of the convex geometry on three colinear points $x, y, z$

an antimatroid is isomorphic to $\mathcal{F}$ ordered by reverse inclusion. If $L$ is a semimodular lattice and $(E, \mathcal{F})$ the interval greedoid described in Proposition 2.2, then the corresponding poset of flats is naturally isomorphic to $L$.

The following result implies that $\Phi$ is a lower semimodular poset. In fact, $\Phi$ is a semimodular lattice; see Proposition 2.9.

Proposition 2.6 [18, Lemma 8.8.6] Suppose $(E, \mathcal{F})$ is an interval greedoid. Let $X \in$ $\mathcal{F}$ and suppose $X \cup x \in \mathcal{F}$ and $X \cup y \in \mathcal{F}$. If $[X \cup x] \neq[X \cup y]$, then $X \cup\{x, y\} \in \mathcal{F}$.

\subsubsection{Maps $\mu$ and $\xi$}

Let $(E, \mathcal{F})$ be an interval greedoid and $\Phi$ its poset of flats. Define two maps $\mu$ : $2^{E} \rightarrow \Phi$ and $\xi: \Phi \rightarrow 2^{E}$ as follows.

( $\mu$ ) Define $\mu: 2^{E} \rightarrow \Phi$ on arbitrary subsets $A \subseteq E$ by $\mu(A)=[X]$, where $X$ is maximal with respect to inclusion among the feasible sets contained in $A$.

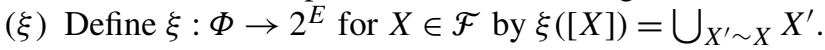

It follows from Proposition 2.4 that $\mu$ is well-defined. These maps are very important to what follows, and will be used to describe the meets and joins in $\Phi$.

Proposition 2.7 Suppose $(E, \mathcal{F})$ is an interval greedoid, $\Phi$ its lattice of flats and $\mu$ and $\xi$ the maps defined above.

(1) $(\mu \circ \xi)(A)=A$ for all $A \in \Phi$. So $\xi$ is injective.

(2) $\mu:\left(2^{E}, \subseteq\right) \rightarrow(\Phi, \leq)$ is order-reversing.

(3) $\xi:(\Phi, \leq) \rightarrow\left(2^{E}, \subseteq\right)$ is order-reversing.

(4) For all $A, B \in \Phi$ we have $A \leq B$ if and only if $\xi(B) \subseteq \xi(A)$.

(5) For all $Y \in \mathcal{F}$ and $A \in \Phi$, if $Y \subseteq \xi(A)$, then $A \leq[Y]$.

Proof (1) Suppose that $X$ is not maximal with respect to inclusion among the feasible sets contained in $\xi([X])$. Then there exists $x \in \xi([X])-X$ such that $X \cup x \in \mathcal{F}$. Therefore, $x \in \mathcal{F} / X$ and $x \in X^{\prime}$ for some $X^{\prime} \sim X$ with $X^{\prime} \neq X$. But $X^{\prime} \sim X$ if 
and only if $\mathcal{F} / X=\mathcal{F} / X^{\prime}$, so $x \in \mathcal{F} / X^{\prime}$. This is a contradiction since $x \notin \mathscr{F} / X^{\prime}$ if $x \in X^{\prime}$. Thus, $X$ is maximal, and so $\mu(\xi([X]))=[X]$.

(2) Suppose $A \subseteq B$. Let $X$ be maximal with respect to inclusion among the feasible sets contained in $A$. Then there exists $Y$ such that $X \subseteq Y \subseteq B$ and $Y$ is maximal among the feasible sets contained in $B$. Therefore, $[Y] \leq[X]$. Hence, $\mu(B) \leq \mu(A)$.

(3) Suppose $[X] \leq[Y]$. If $e \in \xi([Y])$, then $e \in Y^{\prime}$ for some $Y^{\prime} \sim Y$. So $[X] \leq$ $[Y]=\left[Y^{\prime}\right]$. Thus, there is a $Z \in \mathcal{F} / Y^{\prime}$ such that $Y^{\prime} \cup Z \sim X$. Therefore, $e \in Y^{\prime} \subseteq$ $\left(Y^{\prime} \cup Z\right) \subseteq \xi([X])$.

(4) This follows from (1), (2) and (3).

(5) Suppose $Y \in \mathcal{F}$ and $Y \subseteq \xi([X])$. Then there exists $Z$ containing $Y$ that is maximal among the feasible sets contained in $\xi([X])$. Then $[Y] \geq[Z]$ since $Y \subseteq Z$ and $[Z]=[X]$ by Proposition 2.4 .

Remark 2.8 For a matroid without loops, $(\xi \circ \mu)(A)$ is the smallest closed set containing $A$. There is a generalization of the notion of closed sets for any interval greedoid $[18, \S 8.4 \mathrm{~B}]$, and in this generality all one can say is that $(\xi \circ \mu)(A)$ is contained in the smallest closed set containing $A$. That this containment can be strict follows by considering the convex geometry on three colinear points.

For a matroid without loops, $\xi(\Phi)$ consists exactly of the closed sets of the matroid. For an antimatroid, $\xi(\Phi)$ consists precisely of the feasible sets; for the interval greedoid arising from a semimodular lattice, $\xi([X])$ consists of the set of meetirreducibles $f$ such that $f \geq \phi([X])$.

\subsubsection{Lattice of Flats}

We have seen that $\Phi$ is a lower semimodular poset (Proposition 2.6). It is also graded: the corank of any element $A \in \Phi$ is the size of any feasible set in $A$. It turns out that $\Phi$ is also a lattice [18, Theorem 8.8.7]. The next result reformulates the lattice operations in terms of the maps $\mu$ and $\xi$.

Proposition 2.9 If $(E, \mathcal{F})$ is an interval greedoid, then $\Phi$ is a lower semimodular lattice whose lattice operations are given by

$$
A \vee B=\mu(\xi(A) \cap \xi(B)) \text { and } A \wedge B=\mu(\xi(A) \cup \xi(B))
$$

for all $A, B \in \Phi$. That is, $A \vee B=[X]$, where $X$ is maximal among the feasible sets contained in $\xi(A) \cap \xi(B)$, and $A \wedge B=[Y]$, where $Y$ is maximal among the feasible sets contained in $\xi(A) \cup \xi(B)$.

If $(E, \tau)$ is a convex geometry and $(E, \mathcal{F})$ the corresponding antimatroid, then

$$
[X] \vee[Y]=[E \backslash \tau((E \backslash X) \cup(E \backslash Y))]
$$

for all $X, Y \in \mathcal{F}$.

\section{Oriented Interval Greedoids}

Throughout this section $(E, \mathcal{F})$ will denote an interval greedoid. 


\subsection{Signed Flats}

A signed flat of an interval greedoid $(E, \mathcal{F})$ is a pair $(A, \widehat{\alpha})$ consisting of a flat $A$ and a map $\widehat{\alpha}: \Gamma(A) \rightarrow\{+,-\}$.

Define a partial order on signed flats as follows. If $(A, \widehat{\alpha})$ and $(B, \widehat{\beta})$ are signed flats of $(E, \mathcal{F})$, let $(A, \widehat{\alpha}) \leq(B, \widehat{\beta})$ if $A \leq B$ (as flats in $\Phi)$ and if $\widehat{\alpha}$ and $\widehat{\beta}$ agree on $\Gamma(A) \cap \Gamma(B)$. Reflexivity and anti-symmetry are straightforward to verify; transitivity follows from (IG3).

Define the product $(A, \widehat{\alpha}) \circ(B, \widehat{\beta})$ of two signed flats $(A, \widehat{\alpha})$ and $(B, \widehat{\beta})$ by

$$
(A, \widehat{\alpha}) \circ(B, \widehat{\beta})=(A \vee B, \widehat{\alpha} \circ \widehat{\beta}),
$$

where, for $x \in \Gamma(A \vee B)$,

$$
(\widehat{\alpha} \circ \widehat{\beta})(x)= \begin{cases}\widehat{\alpha}(x), & \text { if } x \in \Gamma(A), \\ \widehat{\beta}(x), & \text { otherwise. }\end{cases}
$$

This product is well-defined because $\Gamma(A \vee B) \subseteq \Gamma(A) \cup \Gamma(B)$ by Proposition 3.3 below.

Example 3.1 (Antimatroids) Suppose $(E, \mathcal{F})$ is an antimatroid. The continuations of a feasible set $X$ are the extreme points of the complement $E \backslash X$ in the convex geometry. Therefore, a signed flat $([X], \widehat{\alpha})$ of the antimatroid is an assignment of + or - to each extreme point of $E \backslash X$. Figure 2 depicts a closed set $C$ of a convex geometry; the extreme points of $C$ are labeled by + or - , the non-extreme points in $C$ are labeled by 1 , and the points in the exterior of $C$ are labeled by 0 .

The product of two signed flats $([X], \widehat{\alpha})$ and $([Y], \widehat{\beta})$ has a geometric interpretation. If $X^{\prime}=E \backslash X$ and $Y^{\prime}=E \backslash Y$, then form a new closed set $Z^{\prime}$ by taking the closure of $X^{\prime} \cup Y^{\prime}$; that is, $Z^{\prime}=\tau\left(X^{\prime} \cup Y^{\prime}\right)$. Note that the extreme points of $Z^{\prime}$ are contained in $\operatorname{ext}\left(X^{\prime}\right) \cup \operatorname{ext}\left(Y^{\prime}\right)$. The sign for each $z \in \operatorname{ext}\left(Z^{\prime}\right)$ is $\widehat{\alpha}(z)$ if $z \in \operatorname{ext}\left(X^{\prime}\right)$, and $\widehat{\beta}(z)$ otherwise.

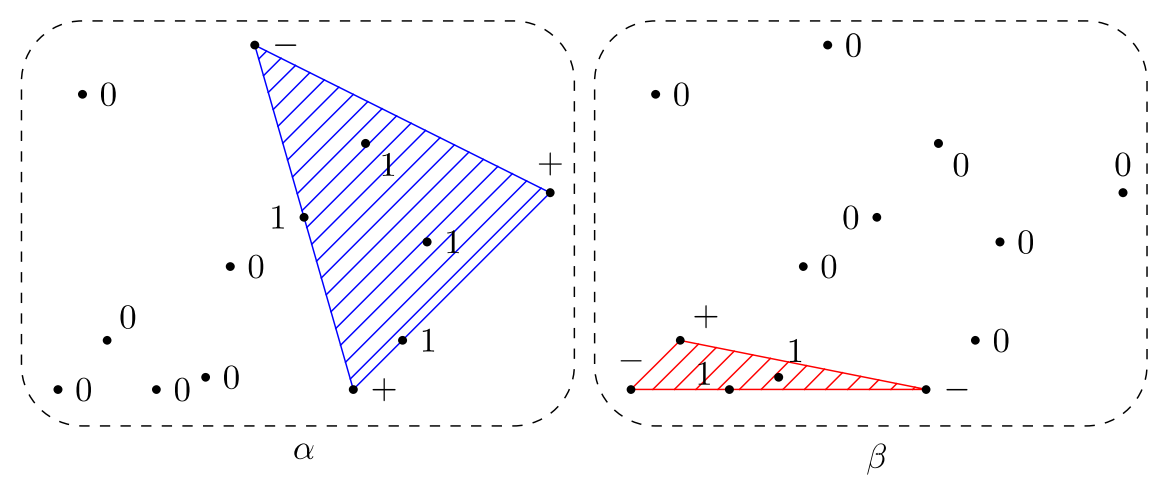

Fig. 2 Two covectors $\alpha$ and $\beta$ of an antimatroid 
Example 3.2 (Matroids) Suppose $(E, \mathcal{F})$ is a matroid with no loops. If $A$ is a flat of the matroid, then $\xi(A)=E \backslash \Gamma(A)$ is a closed set of the matroid. Therefore, a signed flat $(A, \widehat{\alpha})$ is an assignment of a sign + or - to each element of the complement of the closed set $A$. If we extend this by assigning 0 to each element of $A$, then $\widehat{\alpha}$ induces a covector in the sense of oriented matroids (see Sect. 3.4.1).

Among other things, the following establishes that the product of signed flats is well-defined.

Proposition 3.3 Let $(E, \mathcal{F})$ be an interval greedoid, $A, B \in \Phi$ and $X \in \mathcal{F}$.

(1) If $B \leq[X]$ and $x \in \Gamma(X)$, then either $x \in \Gamma(B)$ or $B \leq[X \cup x]$.

(2) If $A \leq B$, then $\Gamma(B) \subseteq \Gamma(A) \cup \xi(A)$.

(3) $\Gamma(A \vee B) \subseteq \Gamma(A) \cup \Gamma(B)$.

(4) $\Gamma(A \vee B) \cup \xi(A \vee B) \subseteq(\Gamma(A) \cup \xi(A)) \cap(\Gamma(B) \cup \xi(B))$.

Proof (1) Pick $Y \in \mathcal{F}$ such that $B=[Y]$. Suppose $[Y] \leq[X]$ and let $x \in \Gamma(X)$. Then there exists $Z \in \mathcal{F} / X$ such that $X \cup Z \sim Y$. Applying axiom (IG2) repeatedly to $X$ and $X \cup Z$ yields a sequence $X \subset\left(X \cup z_{1}\right) \subset\left(X \cup\left\{z_{1}, z_{2}\right\}\right) \subset \cdots \subset(X \cup Z)$ of feasible sets. Put $X_{0}=X$ and let $X_{i}=X_{i-1} \cup z_{i}$ for $1 \leq i \leq r=|Z|$.

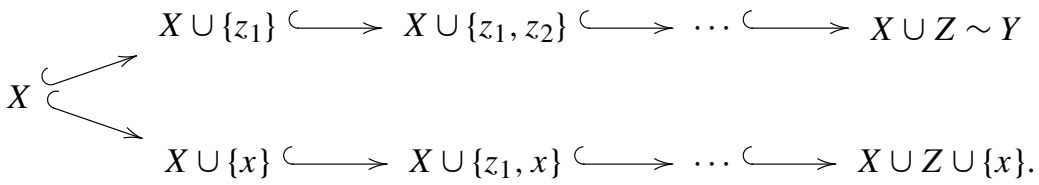

Since $x \in \Gamma(X), X \cup x \in \mathcal{F}$. If $[X \cup x]=\left[X \cup z_{1}\right]$, then $[Y]=[X \cup Z] \leq[X \cup$ $\left.z_{1}\right]=[X \cup x]$ since $X \subseteq X \cup Z$. If $[X \cup x] \neq\left[X \cup z_{1}\right]$, then $X \cup\left\{x, z_{1}\right\} \in \mathcal{F}$ by Proposition 2.6 since $x, z_{1} \in \Gamma(X)$. Thus, $x, z_{2} \in \Gamma\left(X \cup z_{1}\right)$. If $\left[X \cup\left\{z_{1}, x\right\}\right]=[X \cup$ $\left.\left\{z_{1}, z_{2}\right\}\right]$, then $[Y] \leq[X \cup x]$ using a similar argument as in the previous case. If $\left[X \cup\left\{z_{1}, x\right\}\right] \neq\left[X \cup\left\{z_{1}, z_{2}\right\}\right]$, then $X \cup\left\{z_{1}, z_{2}, x\right\} \in \mathcal{F}$ by Proposition 2.6. Continuing in this manner we get either that $[Y] \leq[X \cup x]$ or $(X \cup Z) \cup x \in \mathcal{F}$. That is, either $B=[Y] \leq[X \cup x]$, or $x \in \Gamma(Y)=\Gamma(B)$. This proves the statement.

(2) Pick $X, Y \in \mathcal{F}$ such that $A=[Y]$ and $B=[X]$. If $[Y] \leq[X]$ and $x \in \Gamma(Y)$, then $x \in \Gamma(Y)$ or $[Y] \leq[X \cup x]$ by (1). In the latter case, $x \in \xi(X \cup x) \subseteq \xi(Y)$ since $\xi$ is order-reversing. Thus, $x \in \Gamma(Y)$ or $x \in \xi(Y)$.

(3) Pick $X \in \mathcal{F}$ such that $A \vee B=[X]$. Let $x \in \Gamma(X)$. Then $X \cup x \in \mathcal{F}$ and $[X \cup x]<[X]$. Since $[X]=A \vee B$, it follows that $[X \cup x]$ is not above both $A$ and $B$. If $A \not \leq[X \cup x]$, then the above applied to $A \vee B$ and $A$ gives that $x \in \Gamma(A)$ since $A \not \leq[X \cup x]$. Similarly, if $B \not \leq[X \cup x]$, then $x \in \Gamma(B)$. Hence, $x \in \Gamma(A) \cup \Gamma(B)$.

(4) Since $\xi$ is order-reversing, it follows that $\xi(A \vee B) \subseteq \xi(A) \cap \xi(B)$. (4) now follows from (2).

The following result summarizes properties of the product and partial order.

Proposition 3.4 Let $(A, \widehat{\alpha})$ and $(B, \widehat{\beta})$ denote two signed flats over an interval greedoid $(E, \mathcal{F})$. Then 
(1) $(A, \widehat{\alpha}) \leq(B, \widehat{\beta})$ if and only if $(A, \widehat{\alpha}) \circ(B, \widehat{\beta})=(B, \widehat{\beta})$.

(2) $(A, \widehat{\alpha}) \leq(A, \widehat{\alpha}) \circ(B, \widehat{\beta})$.

(3) If $A \leq \bar{B}$, then $(B, \widehat{\beta}) \circ(A, \widehat{\alpha})=(B, \widehat{\beta})$.

(4) The product $\circ$ is associative.

(5) $(A, \widehat{\alpha}) \circ(B, \widehat{\beta}) \circ(A, \widehat{\alpha})=(A, \widehat{\alpha}) \circ(B, \widehat{\beta})$.

(6) $(A, \widehat{\alpha}) \circ(A, \widehat{\alpha})=(A, \widehat{\alpha})$.

Proof (1) Suppose $(A, \widehat{\alpha}) \circ(B, \widehat{\beta})=(B, \widehat{\beta})$. Since $(A, \widehat{\alpha}) \circ(B, \widehat{\beta})=(A \vee B, \widehat{\alpha} \circ \widehat{\beta})$, it follows that $B=A \vee B$ and that $\widehat{\beta}=\widehat{\alpha} \circ \widehat{\beta}$. Therefore, $A \leq B$ and $\widehat{\beta}(x)=(\widehat{\alpha} \circ$ $\widehat{\beta})(x)=\widehat{\alpha}(x)$ for all $x \in \Gamma(A) \cap \Gamma(B)$. Thus, $(A, \widehat{\alpha}) \leq(B, \widehat{\beta})$.

Conversely, suppose $(A, \widehat{\alpha}) \leq(B, \widehat{\beta})$. Then $A \leq B$ and $\widehat{\alpha}(x)=\widehat{\beta}(x)$ for all $x \in$ $\Gamma(A) \cap \Gamma(B)$. Therefore, $A \vee B=B$. It remains to show that $(\widehat{\alpha} \circ \widehat{\beta})(x)=\widehat{\beta}(x)$ for all $x \in \Gamma(A \vee B)=\Gamma(B)$. Let $x \in \Gamma(B)$. If $x \in \Gamma(A)$, then $(\widehat{\alpha} \circ \widehat{\beta})(x)=\widehat{\alpha}(x)$ and $\widehat{\alpha}(x)=\widehat{\beta}(x)$ since $\widehat{\alpha}$ and $\widehat{\beta}$ agree on $\Gamma(A) \cap \Gamma(B)$. If $x \notin \Gamma(A)$, then $(\widehat{\alpha} \circ \widehat{\beta})(x)=$ $\widehat{\beta}(x)$. Therefore, $\widehat{\beta}$ and $\widehat{\alpha} \circ \widehat{\beta}$ agree on $\Gamma(A \vee B)$.

(2) First note that $A \leq A \vee B$ by the definition of $\vee$. We need only show that $\widehat{\alpha}$ and $\widehat{\alpha} \circ \widehat{\beta}$ agree on $\Gamma(A) \cap \Gamma(A \vee B)$, which follows from the definition of $\widehat{\alpha} \circ \widehat{\beta}$. Therefore, $(A, \widehat{\alpha}) \leq(A \vee B, \widehat{\alpha} \circ \widehat{\beta})=(A, \widehat{\alpha}) \circ(B, \widehat{\beta})$.

(3) If $A \leq B$, then $A \vee B=B$, so $\Gamma(A \vee B)=\Gamma(B)$. So the domains of $\widehat{\beta} \circ \widehat{\alpha}$ and $\widehat{\beta}$ are the same. And from the definition of o, if $x \in \Gamma(B)$, then $(\widehat{\beta} \circ \widehat{\alpha})(x)=\widehat{\beta}(x)$.

(4), (5) and (6) are straightforward to verify using similar arguments.

\subsection{Covectors}

Let $(A, \widehat{\alpha})$ denote a signed flat. Then $\widehat{\alpha}: \Gamma(A) \rightarrow\{+,-\}$ can be extended to a map $\alpha: E \rightarrow\{0,+,-, 1\}$ as follows:

$$
\alpha(e)= \begin{cases}\widehat{\alpha}(e), & \text { if } e \in \Gamma(A), \\ 0, & \text { if } e \in \xi(A), \\ 1, & \text { otherwise. }\end{cases}
$$

This map $\alpha$ is called the covector of the signed flat $(A, \widehat{\alpha})$.

Note that a signed flat $(A, \widehat{\alpha})$ can be recovered from its covector $\alpha$. Indeed, the set of indices $e \in E$ such that $\alpha(e)=0$ is precisely the set $\xi(A)$, from which $A$ can be recovered (Proposition 2.7). Therefore, there exists a map from the set of covectors of $(E, \mathcal{F})$ to the lattice of flats $\Phi$, called the support of the covector,

$$
\operatorname{supp}(\alpha)=\mu(\{x \in E: \alpha(x)=0\}) .
$$

The product on signed flats can be formulated for covectors as follows. Let $\alpha$ and $\beta$ be the covectors of the signed flats $(A, \widehat{\alpha}),(B, \widehat{\beta})$, respectively. Partially order the symbols $0,+,-, 1$ so that 0 and 1 are the (unique) minimal and maximal elements, respectively, and + and - are incomparable. Define

$$
(\alpha \star \beta)(e)= \begin{cases}\beta(e), & \text { if } \beta(e)>\alpha(e), \\ \alpha(e), & \text { otherwise }\end{cases}
$$


and

$$
(\alpha \circ \beta)(e)= \begin{cases}(\alpha \star \beta)(e), & \text { if } e \in \Gamma(A \vee B) \cup \xi(A \vee B), \\ 1, & \text { otherwise }\end{cases}
$$

Proposition 3.5 Suppose $\alpha$ and $\beta$ are the covectors of the signed flats $(A, \widehat{\alpha})$ and $(B, \widehat{\beta})$, respectively. Then the covector $\gamma$ of $(A, \widehat{\alpha}) \circ(B, \widehat{\beta})$ is $\alpha \circ \beta$.

Proof By definition, the covector $\gamma$ of the signed flat $(A \vee B, \widehat{\alpha} \circ \widehat{\beta})$ is given by $\gamma(e)=(\widehat{\alpha} \circ \widehat{\beta})(e)$ if $e \in \Gamma(A \vee B) ; \gamma(e)=0$ if $e \in \xi(A \vee B)$; and $\gamma(e)=1$ otherwise.

Suppose $e \notin \Gamma(A \vee B) \cup \xi(A \vee B)$. By the definition of the product of covectors, $(\alpha \circ \beta)(e)=1$. Hence, $(\alpha \circ \beta)(e)=\gamma(e)$.

Suppose $e \in \xi(A \vee B)$. Then $e \in \xi(A)$ and $e \in \xi(B)$ since $\xi$ is order-reversing. This implies that $\alpha(e)=\beta(e)=0$, hence $(\alpha \circ \beta)(e)=0$. Therefore, $(\alpha \circ \beta)(e)=$ $\gamma(e)$.

Suppose $e \in \Gamma(A \vee B)$. By Proposition 3.3(3), $e \in \Gamma(A) \cup \Gamma(B)$. If $e \in \Gamma(A)$, then $\beta(e) \ngtr \alpha(e)$, so $(\alpha \circ \beta)(e)=\alpha(e)=\widehat{\alpha}(e)$. If $e \notin \Gamma(A)$, then $e \in \Gamma(B) \cap \xi(A)$ and so $\beta(e)>0=\alpha(e)$. Hence, $(\alpha \circ \beta)(e)=\beta(e)=\widehat{\beta}(e)$. Therefore, $(\alpha \circ \beta)(e)=$ $(\widehat{\alpha} \circ \widehat{\beta})(e)$ for all $e \in \Gamma(A \vee B)$.

Example 3.6 (Antimatroids) If $([X], \widehat{\alpha})$ is a signed flat of an upper interval greedoid, then its covector is obtained by assigning $\widehat{\alpha}(x)$ to the extreme points $x \in \operatorname{ext}(E \backslash X)$, 1 to the non-extreme points contained in $E \backslash X$, and 0 to the points in the exterior of $E \backslash X$. Figure 3 depicts the product of the two covectors in Fig. 2.

Let $\alpha$ and $\beta$ be covectors of $(E, \mathcal{F})$. The separation set of $\alpha$ and $\beta$ is

$$
S(\alpha, \beta)=\{e \in E: \alpha(e)=-\beta(e) \in\{+,-\}\} .
$$

(Note that $S(\alpha, \beta) \subseteq \Gamma(\operatorname{supp}(\alpha)) \cap \Gamma(\operatorname{supp}(\beta))$.) The next result develops the relationship between the product, the partial order and the separation set of covectors.

Lemma 3.7 Let $\alpha$ and $\beta$ be covectors of an interval greedoid $(E, \mathcal{F})$.

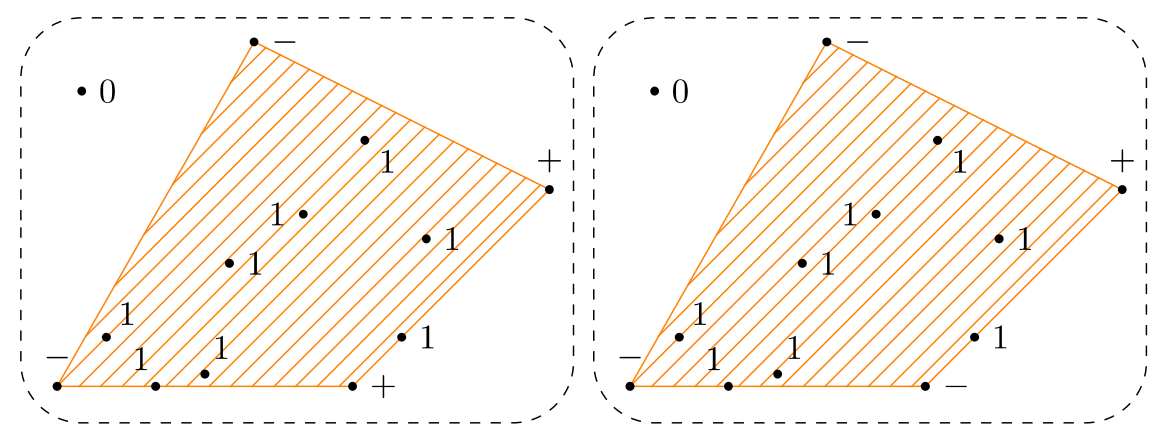

Fig. 3 The products $\alpha \circ \beta$ (left) and $\beta \circ \alpha$ (right) of the covectors $\alpha$ and $\beta$ in Fig. 2 
Fig. 4 Counter-examples to the converse of Lemma 3.7 (4) (cf. Remark 3.8)

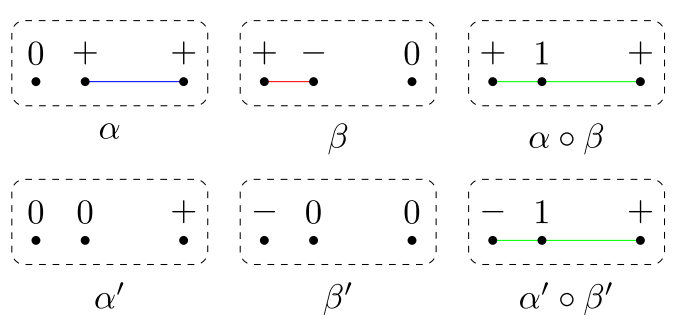

(1) $\alpha \leq \beta$ if and only if $\alpha \circ \beta=\beta$.

(2) $\alpha \leq \beta$ if and only if $\alpha(e) \leq \beta(e)$ for all $e \in E$.

(3) $\alpha \leq \beta$ if and only if $S(\alpha, \beta)=\varnothing$ and $\operatorname{supp}(\alpha) \leq \operatorname{supp}(\beta)$.

(4) If $\alpha(e)=1$ or $\beta(e)=1$, then $(\alpha \circ \beta)(e)=1=(\beta \circ \alpha)(e)$.

Proof Let $A=\operatorname{supp}(\alpha)$ and $B=\operatorname{supp}(\beta)$. By definition, $\alpha \leq \beta$ if and only if $A \leq B$ and $\alpha$ and $\beta$ agree on $\Gamma(A) \cap \Gamma(B)$.

(1) This follows from Proposition 3.4 and Proposition 3.5.

(2) Suppose $\alpha \leq \beta$ and let $e \in E$. If $e \notin \xi(B) \cup \Gamma(B)$, then $\beta(e)=1$, so $\alpha(e) \leq$ $\beta(e)$. If $e \in \xi(A)$, then $\alpha(e)=0$, so $\alpha(e) \leq \beta(e)$. So suppose $e \in \xi(B) \cup \Gamma(B)$ and $e \notin \xi(A)$. Then $e \in \Gamma(A) \cap \Gamma(B)$, by Proposition 3.3. Then $\alpha(e) \leq \beta(e)$ because $\alpha$ and $\beta$ agree on $\Gamma(A) \cap \Gamma(B)$.

Conversely, suppose $\alpha(e) \leq \beta(e)$ for all $e \in E$. Since $\{e: \beta(e)=0\} \subseteq\{e$ : $\alpha(e)=0\}$, we have $\operatorname{supp}(\alpha) \leq \operatorname{supp}(\beta)$. If $e \in \Gamma(A) \cap \Gamma(B)$, then $\alpha(e), \beta(e) \in$ $\{+,-\}$, which implies $\alpha(e)=\beta(e)$ because $\alpha(e) \leq \beta(e)$. Thus, $\alpha \leq \beta$.

(3) If $\alpha \leq \beta$, then $A \leq B$, and $S(\alpha, \beta)=\varnothing$ because $S(\alpha, \beta) \subseteq \Gamma(A) \cap \Gamma(B)$. Conversely, if $A \leq B$ and $S(\alpha, \beta)=\varnothing$, then $\alpha(e)=\beta(e)$ for all $e \in \Gamma(A) \cap \Gamma(B)$, so $\alpha \leq \beta$.

(4) If $(\alpha \circ \beta)(e) \neq 1$, then $e \in \Gamma(A \vee B) \cup \xi(A \vee B) \subseteq(\Gamma(A) \cup \xi(A)) \cap(\Gamma(B) \cup$ $\xi(B))$ by Proposition 3.3(4). Hence, $\alpha(e) \neq 1$ and $\beta(e) \neq 1$.

Remark 3.8 The converse of (4) is false. Counter-examples amongst the covectors of the antimatroid on three colinear points are depicted in Fig. 4. They also illustrate that the following containments can be proper.

$$
\begin{gathered}
\Gamma(A \vee B) \cup \xi(A \vee B) \subseteq(\Gamma(A) \cup \xi(A)) \cap(\Gamma(B) \cup \xi(B)), \\
\xi\left(A^{\prime} \vee B^{\prime}\right) \subseteq \xi\left(A^{\prime}\right) \cap \xi\left(B^{\prime}\right) .
\end{gathered}
$$

\subsection{Oriented Interval Greedoids}

For any covector $\alpha$, let $-\alpha$ be the covector obtained from $\alpha$ by replacing + with and - with + .

Definition 3.9 An oriented interval greedoid is a triple $(E, \mathcal{F}, \mathcal{G})$, where $(E, \mathcal{F})$ is an interval greedoid and $\mathcal{G}$ is a set of covectors of $(E, \mathcal{F})$ satisfying the following axioms. 
(OG1) The map supp : $\mathcal{G} \rightarrow \Phi$ is surjective.

(OG2) If $\alpha \in \mathcal{G}$, then $-\alpha \in \mathcal{G}$.

(OG3) If $\alpha, \beta \in \mathcal{G}$, then $\alpha \circ \beta \in \mathcal{G}$.

(OG4) If $\alpha, \beta \in \mathcal{G}, x \in S(\alpha, \beta)$ and $(\alpha \circ \beta)(x) \neq 1$, then there exists $\gamma \in \mathcal{G}$ such that $\gamma(x)=0$ and for all $y \notin S(\alpha, \beta)$, if $(\alpha \circ \beta)(y) \neq 1$, then $\gamma(y)=(\alpha \circ \beta)(y)=$ $(\beta \circ \alpha)(y)$

Remark 3.10 Note that the set $\mathcal{G}$ with the operation o forms a semigroup: by (OG3), the set $\mathcal{G}$ is closed under $\circ$, and by Proposition 3.4 the operation is associative. This semigroup also satisfies the identities $x^{2}=x$ and $x y x=x y$ for all $x, y \in \mathcal{G}$ (Proposition 3.4). Semigroups satisfying these identities are called left regular bands, which have found several recent applications to computing spectra of random walks [2, 5 , $11,14,15,27]$ and to the study of the descent algebras of finite Coxeter groups [1, 7 , $14,23]$.

As we will see in Sect. 3.4.1, these conditions are modeled on the covector axioms for oriented matroids. In the next section we will present various examples of oriented interval greedoids. We record here the following observation.

Lemma 3.11 Suppose $\alpha$ and $\beta$ are covectors of an oriented interval greedoid. If $(\alpha \circ \beta)(y) \neq(\beta \circ \alpha)(y)$, then $\alpha(y)=-\beta(y) \in\{+,-\}$ (that is, $y \in S(\alpha, \beta))$.

Proof Let $C=\operatorname{supp}(\alpha \circ \beta)=\operatorname{supp}(\beta \circ \alpha)$. Then $(\alpha \circ \beta)(y)=1$ iff $y \notin \Gamma(C) \cup \xi(C)$ iff $(\beta \circ \alpha)(y)=1$. Similarly, $(\alpha \circ \beta)(y)=0$ iff $y \in \xi(C)$ iff $(\beta \circ \alpha)(y)=0$. Thus $\alpha(y), \beta(y) \subset\{+,-\}$. The result follows.

\subsection{Examples}

This section presents examples of oriented interval greedoids.

\subsubsection{Oriented Matroids}

Let $E$ be a finite set. An oriented matroid is a collection $\mathcal{O}$ of maps from $E$ to $\{0,+,-\}$ that satisfies the following axioms.

(OM1) $\mathcal{O}$ contains the map $z(e)=0$ for all $e \in E$.

(OM2) If $\alpha \in \mathcal{O}$, then $-\alpha \in \mathcal{O}$.

(OM3) If $\alpha, \beta \in \mathcal{O}$, then $\alpha \circ \beta \in \mathcal{O}$, where

$$
(\alpha \circ \beta)(e)= \begin{cases}\alpha(e), & \text { if } \alpha(e) \neq 0, \\ \beta(e), & \text { if } \alpha(e)=0 .\end{cases}
$$

(OM4) Suppose $\alpha, \beta \in \mathcal{O}$ and let $S(\alpha, \beta)=\{e \in E: \alpha(e)=-\beta(e) \neq 0\}$. For every $e \in S(\alpha, \beta)$ there exists $\gamma \in \mathcal{O}$ with $\gamma(e)=0$ and $\gamma(f)=(\alpha \circ \beta)(f)=(\beta \circ$ $\alpha)(f)$ for all $f \notin S(\alpha, \beta)$. 
If $\mathcal{O}$ is an oriented matroid, then the set of zeros of the elements of $\mathcal{O}$ form the closed sets of a matroid $(E, \mathcal{F})$. The matroid $(E, \mathcal{F})$ is the underlying matroid of the oriented matroid and $\mathcal{O}$ is said to be an oriented matroid on $(E, \mathcal{F})$.

Theorem 3.12 Suppose $(E, \mathcal{F})$ is a matroid without loops. Then $\mathcal{O}$ is an oriented matroid with underlying matroid $(E, \mathcal{F})$ if and only if $(E, \mathcal{F}, \mathcal{O})$ is an oriented interval greedoid.

Proof Let $(E, \mathcal{F})$ be a matroid and let $(E, \mathcal{F}, \mathcal{G})$ be an oriented interval greedoid. Since $\xi(A)=E \backslash \Gamma(A)$ for any flat $A$ of a matroid without loops, a covector of $(E, \mathcal{F})$ takes values in $\{0,+,-\}$. Therefore, $\mathcal{G}$ is a collection of maps from $E$ to $\{0,+,-\}$, and $\mathcal{G}$ satisfies (OM1)-(OM4) since it satisfies (OG1)-(OG4). So $\mathcal{G}$ is an oriented matroid.

Conversely, suppose that $\mathcal{O}$ is an oriented matroid with underlying matroid $(E, \mathcal{F})$. If $\alpha \in \mathcal{O}$, then the set $\zeta(\alpha)$ of zeros of $\alpha$ is a closed set of the matroid, and there is a unique flat $A$ satisfying $\xi(A)=\zeta(\alpha)$. Therefore, $\alpha$ gives a signed flat $\left(A,\left.\alpha\right|_{\Gamma(A)}\right)$, and the covector of this signed flat is $\alpha$. So $\mathcal{O}$ is a set of covectors of the interval greedoid $(E, \mathcal{F})$. It is straightforward to check that the axioms for an oriented interval greedoid are satisfied by $\mathcal{O}$.

\subsubsection{Antimatroids}

Next we show that the set of all covectors of an antimatroid forms an oriented interval greedoid. This collection of covectors, viewed as a poset, is the central object of study in the work of Billera, Hsiao, and Provan [4]. We also show that this is the only oriented interval greedoid structure on an antimatroid.

Example 3.13 If $\alpha$ and $\beta$ are the covectors of Fig. 2, then the separation set $S(\alpha, \beta)$ consists of the vertex $x$ circled in Fig. 5. The covector $\gamma$ depicted in Fig. 5 satisfies (OG4) for $\alpha, \beta$ and $x \in S(\alpha, \beta)$.

Theorem 3.14 Suppose $(E, \mathcal{F})$ is an upper interval greedoid. Let $\mathcal{G}$ denote the set of all covectors of $(E, \mathcal{F})$. Then $(E, \mathcal{F}, \mathcal{G})$ is an oriented interval greedoid.

Proof We will show that (OG1)-(OG4) hold.

Fig. 5 If $\alpha$ and $\beta$ are the two covectors in Fig. 2 and $x$ is the circled vertex, then the covector $\gamma$ illustrated here satisfies the conditions of (OG4)

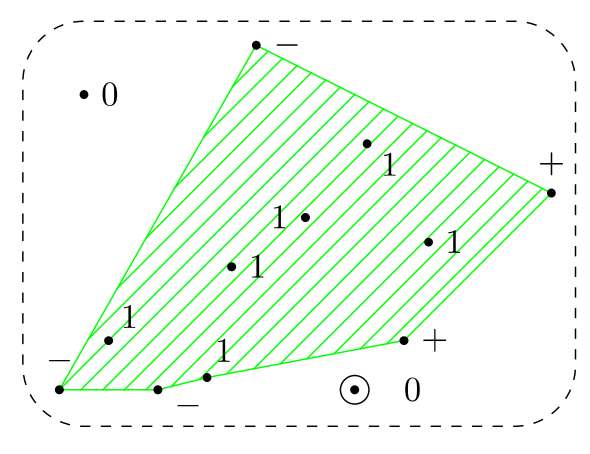


(OG1) Suppose $A \in \Phi$ is a flat. Let $(A, \widehat{\alpha})$ be a signed flat of $(E, \mathcal{F})$ and let $\alpha$ denote the covector of this signed flat. Then $\alpha \in \mathcal{G}$ and $\operatorname{supp}(\alpha)=A$.

(OG2) Suppose $\alpha \in \mathcal{G}$. Let $A=\operatorname{supp}(\alpha)$. Then $\left(A,-\left.\alpha\right|_{\Gamma(A)}\right)$ is a signed flat of $(E, \mathcal{F})$. The covector of this signed flat is precisely $-\alpha$. So $-\alpha \in \mathcal{G}$.

(OG3) If $\alpha, \beta \in \mathcal{G}$, then $\alpha \circ \beta$ is a covector of $(E, \mathcal{F})$, so $\alpha \circ \beta \in \mathcal{G}$.

(OG4) Suppose $\alpha, \beta \in \mathcal{G}$ and $x \in S(\alpha, \beta)$ satisfies $(\alpha \circ \beta)(x) \neq 1$. Let $(E, \tau)$ denote the convex geometry that is complementary to $(E, \mathcal{F})$ (see Sect. 2.1.2). Let $A=\operatorname{supp}(\alpha)$ and $B=\operatorname{supp}(\beta)$. Then $A=[X]$ and $B=[Y]$ for some $X, Y \in \mathcal{F}$. Let $X^{\prime}=E \backslash X$ and $Y^{\prime}=E \backslash Y$. Then $X^{\prime}$ and $Y^{\prime}$ are closed sets in $(E, \tau)$.

Step 1: We show that $x$ is an extreme point of $\tau\left(X^{\prime} \cup Y^{\prime}\right)$. Since $(\alpha \circ \beta)(x) \notin\{0,1\}$, we have $x \in \Gamma(A \vee B)$. Since $A \vee B=\left[E \backslash \tau\left(X^{\prime} \cup Y^{\prime}\right)\right]$ by (2.1), we have $\Gamma(A \vee B)=$ $\operatorname{ext}\left(\tau\left(X^{\prime} \cup Y^{\prime}\right)\right)$. Thus, $x \in \operatorname{ext}\left(\tau\left(X^{\prime} \cup Y^{\prime}\right)\right)$.

Step 2: We define $\gamma$. Let $Z^{\prime}=\tau\left(X^{\prime} \cup Y^{\prime}\right)-x$. Since $x$ is an extreme point of $\tau\left(X^{\prime} \cup Y^{\prime}\right)$, it follows that $x$ is not in $Z^{\prime}$. Hence $Z^{\prime}$ is a closed set not containing $x$. Let $Z=E \backslash Z^{\prime}$. Then $Z \in \mathcal{F}$ and $x \in Z$. Define a map $\gamma^{\prime}: \Gamma(Z) \rightarrow\{+,-\}$ for $y \in \Gamma(Z)$ as follows: if $y \in \Gamma(A \vee B)$, then set $\gamma^{\prime}(y)=(\alpha \circ \beta)(y)$; otherwise arbitrarily set $\gamma^{\prime}(y)$ to be + or - . Let $\gamma$ be the covector of the signed flat $\left([Z], \gamma^{\prime}\right)$.

Step 3: $\gamma$ has the desired properties. First note that $\gamma \in \mathcal{G}$ since $\gamma$ is a covector of $(E, \mathcal{F})$. Next observe that $\gamma(x)=0$ since $x \in Z \subseteq \xi([Z])$. Let $y \notin S(\alpha, \beta)$.

Suppose that $(\alpha \circ \beta)(y) \neq 1$. Then $y \in \Gamma(A \vee B) \cup \xi(A \vee B)$. Since $\xi(A \vee B)=$ $E \backslash \tau\left(X^{\prime} \cup Y^{\prime}\right) \subseteq Z \subseteq \xi([Z])$, if $y \in \xi(A \vee B)$, then $\gamma(y)=0=(\alpha \circ \beta)(y)=(\beta \circ$ $\alpha)(y)$. On the other hand, if $y \in \Gamma(A \vee B)=\operatorname{ext}\left(\tau\left(X^{\prime} \cup Y^{\prime}\right)\right)$, then $y$ is an extreme point of $Z^{\prime}=\tau\left(X^{\prime} \cup Y^{\prime}\right)-x$ (since $y \neq x$ ). Equivalently, $y \in \Gamma(Z)$. So, by definition of $\gamma$ and because $y \notin S(\alpha, \beta), \gamma(y)=(\alpha \circ \beta)(y)=(\beta \circ \alpha)(y)$.

Proposition 3.15 Let $(E, \mathcal{F})$ be an antimatroid. Then the only oriented structure on $(E, \mathcal{F})$ is that constructed in Theorem 3.14.

Proof Let $(E, \mathcal{F}, \mathcal{G})$ be an oriented interval greedoid. Let $\alpha$ be an arbitrary covector. We wish to show that $\alpha \in \mathcal{G}$.

Let $\Gamma(\operatorname{supp}(\alpha))=X=\left\{x_{1}, \ldots, x_{r}\right\}$. Let $Y=E \backslash(X \cup \operatorname{supp}(\alpha))$. For any $x \in X$, $Y_{x}=\operatorname{supp}(\alpha) \cup(X \backslash\{x\})$ is feasible. Also, $\Gamma\left(Y_{x}\right) \cap \Gamma(\operatorname{supp}(\alpha))=\{x\}$. By (OIG1), we can find a covector $\beta_{x} \in \mathcal{G}$ with $\operatorname{supp}\left(\beta_{x}\right)=Y_{x}$. By (OIG2), we can choose $\beta_{x}$ so that $\beta_{x}$ agrees with $\alpha$ on $x$. Now $\beta_{x_{1}} \circ \cdots \circ \beta_{x_{r}}=\alpha$ is in $\mathcal{G}$.

\subsubsection{Complexified Hyperplane Arrangements}

An (essential) real hyperplane arrangement is a finite set of hyperplanes $\left\{\Theta_{1}, \Theta_{2}\right.$, $\left.\ldots, \Theta_{n}\right\}$ in $\mathbb{R}^{d}$ satisfying $\bigcap \Theta_{i}=\{\overrightarrow{0}\}$. Let $E=\{1,2, \ldots, n\}$ and for each $e \in E$ fix a linear form $\ell_{e}: \mathbb{R}^{d} \rightarrow \mathbb{R}$ such that $\Theta_{e}=\operatorname{ker}\left(\ell_{e}\right)$. Extending scalars, we can also think of $\ell_{e}$ as defining a linear map from $\mathbb{C}^{d}$ to $\mathbb{C}$. Define $H_{e}$ to be the kernel of this map. It is a hyperplane in $\mathbb{C}^{d}$. The collection $\mathcal{A}=\left\{H_{1}, \ldots, H_{n}\right\}$ forms a complexified hyperplane arrangement. Also define $H_{e}^{\Re}=\left\{\vec{z} \in \mathbb{C}^{d}: \Im\left(\ell_{e}(\vec{z})\right)=0\right\}$.

(Note that not all complex hyperplane arrangements are complexified arrangements; that is to say, not all complex hyperplane arrangements arise from a real hyperplane arrangement in the way we have just described.) 
For any $z=x+i y \in \mathbb{C}$, let

$$
\sigma_{\mathfrak{R}}(x+i y)=\left\{\begin{array}{ll}
1, & \text { if } y \neq 0, \\
+, & \text { if } y=0, x>0, \\
-, & \text { if } y=0, x<0, \\
0, & \text { if } y=0, x=0,
\end{array} \quad \sigma_{\Im}(x+i y)= \begin{cases}+, & \text { if } y>0, \\
-, & \text { if } y<0, \\
0, & \text { if } y=0,\end{cases}\right.
$$

and for every $\vec{z} \in \mathbb{C}^{d}$, let

$$
\alpha_{\vec{z}}(h)= \begin{cases}\sigma_{\Im}\left(\ell_{i}(\vec{z})\right), & \text { if } h=H_{i}^{\Re}, \\ \sigma_{\Re}\left(\ell_{i}(\vec{z})\right), & \text { if } h=H_{i} .\end{cases}
$$

Note that $\left(\alpha\left(H_{i}\right), \alpha\left(H_{i}^{\Re}\right)\right) \in\{(0,0),(+, 0),(-, 0),(1,+),(1,-)\}$ for all $1 \leq e \leq n$.

Example 3.16 There is a unique complexified hyperplane arrangement in $\mathbb{C}$, namely $\mathcal{A}=\left\{H_{0}=\{\overrightarrow{0}\}\right\}$. In this case

$$
\left\{\alpha_{\vec{z}}: \vec{z} \in \mathbb{C}\right\}=\{(0,0),(+, 0),(-, 0),(1,+),(1,-)\}
$$

is the set of covectors of the interval greedoid on the ground set $E=\left\{H_{0}, H_{0}^{\Re}\right\}$ with feasible sets $\mathcal{F}=\left\{\varnothing,\left\{H_{0}^{\Re}\right\},\left\{H_{0}, H_{0}^{\Re}\right\}\right\}$. Figure 6 illustrates the partial order on these covectors.

Let $\mathcal{A}=\left\{H_{1}, \ldots, H_{n}\right\}$ be a complexified hyperplane arrangement in $\mathbb{C}^{d}$. Let $\mathcal{L}$ be the lattice of all intersections of subspaces from the set

$$
E_{\mathcal{A}}=\left\{H_{1}, \ldots, H_{n}, H_{1}^{\Re}, \ldots, H_{n}^{\Re}\right\},
$$

ordered by inclusion. Then $\mathcal{L}$ is a lower semimodular lattice and $E_{\mathcal{A}}$ is the set of meet-irreducible elements of $\mathcal{L}$ [18]. By Proposition 2.2, $\left(E_{\mathcal{A}}, \mathcal{F}_{\mathcal{A}}\right)$ is an interval greedoid, where

$$
\mathcal{F}_{\mathcal{A}}=\left\{\left\{h_{1}, h_{2}, \ldots, h_{k}\right\} \subseteq E_{\mathcal{A}}: \mathbb{C}^{d}>h_{1}>\cdots>\left(h_{1} \cap h_{2} \cap \cdots \cap h_{k}\right)\right\}
$$

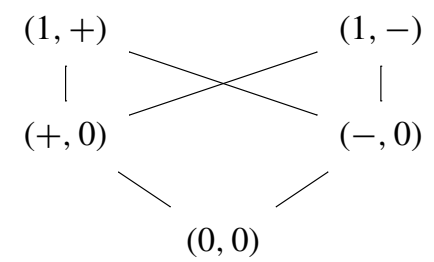

Fig. 6 The poset of covectors of the complex hyperplane arrangement in $\mathbb{C}$ 
Lemma 3.17 Let $\mathcal{A}$ be a complexified hyperplane arrangement, and let $\left(E_{\mathcal{A}}, \widetilde{F}_{\mathcal{A}}\right)$ be the interval greedoid as defined above. Then, for $X \in \mathcal{F}_{\mathcal{A}}$, we have

$$
\begin{gathered}
\xi(X)=\left\{h \in E: \bigcap_{h^{\prime} \in X} h^{\prime} \subseteq h\right\}, \\
\Gamma(X)=\left\{H_{e}^{\Re} \in E: \bigcap_{h \in X} h \nsubseteq H_{e}^{\Re}\right\} \cup\left\{H_{e} \in E: \bigcap_{h \in X} h \subseteq H_{e}^{\Re} \text { and } \bigcap_{h \in X} h \nsubseteq H_{e}\right\} .
\end{gathered}
$$

Proof We prove the first identity holds for any interval greedoid $(E, \mathcal{F})$ constructed from a semimodular lattice $L$ as in Sect. 2.1.3. The poset of flats $\Phi$ of $(E, \mathcal{F})$ is naturally isomorphic to $L$ : the mapping $\phi(X)=\bigwedge_{e \in X} e$ from $\mathcal{F}$ to $L$ descends to a poset isomorphism from $\Phi$ to $L$ (see [19, Theorem 8.8.7] for details). If $X$ is a feasible set, then $\xi([X])$ consists of the set of meet-irreducibles $f$ such that $f \geq \phi([X])$. Indeed, if $f$ is in a feasible set $Y \sim X$, then $\phi([X])=\phi([Y]) \leq f$, which proves one containment. For the other direction, let $f \geq \phi([X])$ and let $Z$ be a feasible set with $\phi([Z])=f$. Since $f$ is meet-irreducible, $f \in Z$. Since $f \geq \phi([X])$, we know $[Z] \geq[X]$, which implies that $f \in Z \subset \xi([X])$, as desired.

We now prove the second identity. Let $M=\bigcap_{h \in X} h \subset \mathbb{C}^{d}$. Thinking of $\mathbb{C}^{d}$ as a $2 d$-dimensional real vector space, we can decompose it into real and complex parts as $\mathbb{C}^{d}=\Re\left(\mathbb{C}^{d}\right) \oplus \Im\left(\mathbb{C}^{d}\right)$, where each of the summands is a $d$-dimensional real vector space, and multiplication by $i$ provides an isomorphism from $\Re\left(\mathbb{C}^{d}\right)$ to $\Im\left(\mathbb{C}^{d}\right)$. Note that $H_{i}$ and $H_{i}^{\Re}$ can also be expressed as a direct sum of a real and an imaginary part. (This relies on the fact that our arrangement is a complexified real arrangement, rather than being an arbitrary complex arrangement.) Note further that in either case, the imaginary part corresponds to a subspace of the real part under the map from $\Re\left(\mathbb{C}^{d}\right)$ to $\Im\left(\mathbb{C}^{d}\right)$. It follows that $M$, also, can be written as $M=\Re(M) \oplus \Im(M)$, with $\Im(M)$ a subspace of $\Re(M)$.

Observe first that if $H_{e}^{\Re} \nsucceq M$, then, since $H_{e}^{\Re}$ is real codimension one in $\mathbb{C}^{d}$, we have $M>M \cap H_{e}^{\Re}$, so $H_{e}^{\Re} \in \Gamma(X)$. Also, in this case, we have $M \cap H_{e}^{\Re}>M \cap H_{e}$, because $\Theta_{e} \geq \Im(M)$, and thus $\Theta_{e} \nsucceq \Re(M)$ either. It follows that in this case $H_{e}^{\Re} \in$ $\Gamma(X)$ and $H_{e} \notin \Gamma(X)$.

Finally, if $H_{e}^{\Re} \geq M$, we observe that $H_{e}$ is codimension one in $H_{e}^{\Re}$, and thus that either $H_{e} \geq M$ or $M>M \cap H_{e}$. This completes the proof of the lemma.

Lemma 3.18 Let $\mathcal{A}=\left\{H_{1}, \ldots, H_{n}\right\}$ be a complexified hyperplane arrangement in $\mathbb{C}^{d}$. Then $\alpha_{\vec{z}}$ is a covector over the interval greedoid $\left(E_{\mathcal{A}}, \mathcal{F}_{\mathcal{A}}\right)$, for every $\vec{z} \in \mathbb{C}^{d}$.

Proof Recall that a map $\alpha: E \rightarrow\{0,+,-, 1\}$ is a covector of an interval greedoid $(E, \mathcal{F})$ if and only if there exists $X \in \mathcal{F}$ such that $\alpha(e)=0$ if and only if $e \in \xi(X)$, $\alpha(e)= \pm$ if and only if $e \in \Gamma(X)$, and $\alpha(e)=1$ otherwise.

Let $\alpha=\alpha_{\vec{z}}$ be defined as above. Observe that $\alpha(h)=0$ if and only if $\vec{z} \in h$. Let

$$
A=\{h \in E: \alpha(h)=0\}=\left\{H_{i}: \vec{z} \in H_{i}\right\} \cup\left\{H_{i}^{\Re}: \vec{z} \in H_{i}^{\Re}\right\} \subseteq E
$$

and let $X$ be maximal among the elements of $\mathcal{F}_{\mathcal{A}}$ contained in $A$. 
We show that $\alpha(h)=0$ if and only if $h \in \xi(X)$ by showing that $\xi(X)=A$. Suppose $h \in \xi(X)$. Then $h \supseteq \bigcap_{h^{\prime} \in X} h^{\prime}$. Since $X \subseteq A$, it follows that $\alpha\left(h^{\prime}\right)=0$ for all $h^{\prime} \in X$. Thus, $\vec{z} \in h^{\prime}$ for all $h^{\prime} \in X$. It follows that $\vec{z} \in h$. Thus, $h \in A$.

Conversely, suppose $h \in A$. Then $\alpha(h)=0$. If $h=H_{i}^{\Re}$, then $\{h\} \in \mathcal{F}_{\mathcal{A}}$, so we can augment $\{h\}$ from $X$ until we get a set $Y$ of cardinality $|X|$. Since $X$ is maximal among the feasible sets contained in $A$ and $|X|=|Y|, Y$ is maximal as well. Thus, $X \sim Y$, so $Y \subseteq \xi(X)$. In particular, $h \in \xi(X)$. On the other hand, if $h=H_{i}$, then $\ell_{i}(\vec{z})=0$, so $H_{i}^{\Re} \in A$ also. Since $\left\{H_{i}, H_{i}^{\Re}\right\} \in \mathcal{F}_{\mathcal{A}}$, the same argument shows that $h \in \xi(X)$. Thus, $A \subseteq \xi(X)$.

Next we show that $\alpha(h) \in\{+,-\}$ if and only if $\alpha(h) \in \Gamma(X)$. Let $h \in \Gamma(X)$. Since $\xi(X)$ and $\Gamma(X)$ are disjoint, it follows from the above that $\alpha(h) \neq 0$. So it suffices to show that $\alpha(h) \in\{0,+,-\}$. By construction, this is true for $h=H_{i}^{\Re}$ since $\alpha\left(H_{i}^{\Re}\right)=\sigma_{\Im}\left(\ell_{i}(\vec{z})\right) \in\{0,+,-\}$. If $h=H_{i}$, then, by the above description of $\Gamma(X)$, we have $H_{i}^{\Re} \in \xi(X)$. So, $\sigma_{\Im}\left(\ell_{i}(\vec{z})\right)=0$, which implies $\alpha(h)=\sigma_{\mathfrak{R}}\left(\ell_{i}(\vec{z})\right) \in\{0,+,-\}$.

Conversely, suppose $\alpha(h) \in\{+,-\}$. Since $\alpha(h) \neq 0$, we have $h \notin \xi(X)$, or equivalently, $\bigcap_{x \in X} x \nsubseteq h$. So if $h=H_{i}^{\Re}$, then $h \in \Gamma(X)$. If $h=H_{i}$, then we need to show that $H_{i}^{\Re} \supseteq \bigcap_{x \in X} x$, or equivalently, $H_{i}^{\Re} \in \xi(X)$. Well, $\sigma_{\Re}\left(\ell_{i}(\vec{z})\right)=\alpha\left(H_{i}\right) \in\{+,-\}$, so $\sigma_{\Im}\left(\ell_{i}(\vec{z})\right)=0$. This implies $H_{i}^{\Re} \in \xi(X)$. Hence, $h=H_{i} \in \Gamma(X)$.

Finally, it follows from the above that $\alpha(h)=1$ if and only if $h \notin \Gamma(X) \cup \xi(X)$. Therefore, $\alpha$ is a covector of $\left(E_{\mathcal{A}}, \mathcal{F}_{\mathcal{A}}\right)$.

Remark 3.19 The product of two covectors $\alpha$ and $\beta$ can also be computed as

$$
\begin{aligned}
& \left((\alpha \circ \beta)\left(H_{i}\right),(\alpha \circ \beta)\left(H_{i}^{\Re}\right)\right) \\
& \quad= \begin{cases}\left(\beta\left(H_{i}\right), \beta\left(H_{i}^{\Re}\right)\right), & \text { if }\left(\beta\left(H_{i}\right), \beta\left(H_{i}^{\Re}\right)\right)>\left(\alpha\left(H_{i}\right), \alpha\left(H_{i}^{\Re}\right)\right), \\
\left(\alpha\left(H_{i}\right), \alpha\left(H_{i}^{\Re}\right)\right), & \text { otherwise, }\end{cases}
\end{aligned}
$$

where the comparison $\left(\beta\left(H_{i}\right), \beta\left(H_{i}^{\Re}\right)\right)>\left(\alpha\left(H_{i}\right), \alpha\left(H_{i}^{\Re}\right)\right)$ is performed in the poset illustrated in Fig. 6.

Theorem 3.20 If $\mathcal{A}=\left\{H_{1}, \ldots, H_{n}\right\}$ is a complexified hyperplane arrangement in $\mathbb{C}^{d}$, then $\mathcal{G}=\left\{\alpha_{\vec{z}}: \vec{z} \in \mathbb{C}^{d}\right\}$ is an oriented interval greedoid over $\left(E_{\mathcal{A}}, \mathcal{F}_{\mathcal{A}}\right)$.

Proof We show that $\mathcal{G}$ satisfies (OG1)-(OG4).

(OG1) Suppose $[X]$ is a flat of $\left(E_{\mathcal{A}}, \mathcal{F}_{\mathcal{A}}\right)$ for some $X \in \mathcal{F}_{\mathcal{A}}$. Let $\vec{z}$ be a generic point of $\bigcap_{x \in X} x$. The support of $\alpha_{\vec{z}}$ is the flat $[Y]$ such that $Y$ is maximal among feasible sets contained in $\left\{h: \alpha_{\vec{z}}(h)=0\right\}$. Since $\vec{z}$ is generic, this set is equal to $\xi(X)$. It follows that $Y$ is equivalent to $X$, so $[X]=[Y]=\operatorname{supp}\left(\alpha_{\vec{z}}\right)$.

(OG2) Suppose $\alpha_{\vec{z}} \in \mathcal{G}$. Then $-\alpha_{\vec{z}}=\alpha_{-\vec{z}}$ because $\sigma_{i}\left(\ell_{j}(-\vec{z})\right)=\sigma_{i}\left(-\ell_{j}(\vec{z})\right)$. So $-\alpha_{\vec{z}} \in \mathcal{G}$.

(OG3) Let $\alpha_{\vec{x}}, \alpha_{\vec{y}} \in \mathcal{G}$. For sufficiently small $t>0$, we have $\sigma_{\Re}(\vec{u}+t \vec{v})=$ $\sigma_{\Re}(\vec{u}) \star \sigma_{\Re}(\vec{v})$ and $\sigma_{\Im}(\vec{u}+t \vec{v})=\sigma_{\Im}(\vec{u}) \star \sigma_{\Im}(\vec{v})$. It follows that $\alpha_{\vec{x}+t \vec{y}}=\alpha_{\vec{x}} \circ \alpha_{\vec{y}}$ for a sufficiently small $t>0$.

(OG4) Let $\alpha, \beta \in \mathcal{G}$ and $h \in S(\alpha, \beta)$ such that $(\alpha \circ \beta)(h) \neq 1$. Pick $\vec{x}, \vec{y} \in \mathbb{C}^{d}$ such that $\alpha=\alpha_{\vec{x}}$ and $\beta=\alpha_{\vec{y}}$. We can assume for all $1 \leq i \leq n$ that the line $t \vec{x}+(1-t) \vec{y}$, 
for $0<t<1$, does not intersect $H_{i}$ if $\vec{x}$ and $\vec{y}$ are not both contained in $H_{i}^{\Re}$ (otherwise perturb $\vec{x}$ and $\vec{y}$ slightly).

Since $h \in S\left(\alpha_{\vec{x}}, \alpha_{\vec{y}}\right)$, we have $\alpha_{\vec{x}}(h)=-\alpha_{\vec{y}}(h) \in\{+,-\}$. Hence, $\Re\left(\ell_{h}(\vec{x})\right)$ and $\Re\left(\ell_{h}(\vec{y})\right)$ or $\Im\left(\ell_{h}(\vec{x})\right)$ and $\Im\left(\ell_{h}(\vec{y})\right)$ have opposite signs, where $\ell_{h}$ is the form associated to $h$ (that is, $h=\operatorname{ker}\left(\ell_{h}\right)$ or $\left.h=\operatorname{ker}\left(\ell_{h}\right)^{\Re}\right)$. So there exists $0<t<1$ such that

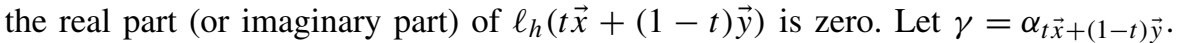
Then $\gamma(h)=0$.

Let $e \notin S\left(\alpha_{\vec{x}}, \alpha_{\vec{y}}\right)$ and $\left(\alpha_{\vec{x}} \circ \alpha_{\vec{y}}\right)(e) \neq 1$. Suppose first that $e=H_{i}$ for some $i$. Then $\Im\left(\ell_{i}(\vec{x})\right)=0=\Im\left(\ell_{i}(\vec{y})\right)$, for otherwise $\left(\alpha_{\vec{x}} \circ \alpha_{\vec{y}}\right)(e)=1$. This implies that $\Im\left(t \ell_{i}(\vec{x})+(1-t) \ell_{i}(\vec{y})\right)=0$, so $\gamma(e)=\sigma_{\Re}\left(\ell_{i}(t \vec{x}+(1-t) \vec{y})\right)$ is the sign of

$$
\Re\left(\ell_{i}(t \vec{x}+(1-t) \vec{y})\right)=t \Re\left(\ell_{i}(\vec{x})\right)+(1-t) \Re\left(\ell_{i}(\vec{y})\right) .
$$

Since both of the coefficients $t$ and $(1-t)$ are positive and since $\Re\left(\ell_{i}(\vec{x})\right)$ and $\Re\left(\ell_{i}(\vec{y})\right)$ are not of opposite signs, it follows that $\gamma(e)$ is the sign of $\Re\left(\ell_{i}(\vec{x})\right)$ if it is nonzero and the sign of $\Re\left(\ell_{i}(\vec{y})\right)$ otherwise. This is precisely $\left(\alpha_{\vec{x}} \circ \alpha_{\vec{y}}\right)(e)$. Similarly, if $e=H_{i}^{\Re} \notin S\left(\alpha_{\vec{x}}, \alpha_{\vec{y}}\right)$, then $\gamma(e)=\left(\alpha_{\vec{x}} \circ \alpha_{\vec{y}}\right)(e)$.

\section{Restriction and Contraction of Oriented Interval Greedoids}

\subsection{Contraction}

This section introduces an operation on oriented interval greedoids that produces an oriented interval greedoid on the contraction of the underlying interval greedoid. We begin by studying the relationship between an interval greedoid and its contractions.

\subsubsection{Contraction of Interval Greedoids}

Let $(E, \mathcal{F})$ denote an interval greedoid and $\Phi$ its lattice of flats. Recall that for $X \in \mathcal{F}$, the contraction of $(E, \mathcal{F})$ by $X$ is the interval greedoid with feasible sets

$$
\mathcal{F} / X=\{Y \subseteq E \backslash X: Y \cup X \in \mathcal{F}\}
$$

and ground set $\bigcup_{Y \in \mathcal{F} / X} Y$. We let $\Phi / X, \Gamma / X$ and $\xi / X$ denote the corresponding notions in the contraction. For $Y \in \mathcal{F} / X$, we let $(\Phi / X)(Y)$ denote the flat in the contraction that contains $Y$.

Proposition 4.1 Suppose $(E, \mathcal{F})$ is an interval greedoid and $X \in \mathcal{F}$. Then

(1) $\Phi / X \cong[\hat{0},[X]] \subseteq \Phi$.

(2) If $Y \in \mathcal{F} / X$, then $(\Gamma / X)(Y)=\Gamma(X \cup Y)$.

(3) If $Y \in \mathcal{F} / X$, then $(\xi / X)(Y) \subseteq \xi(X \cup Y) \cap \bigcup_{Z \in \mathcal{F} / X} Z$.

Proof (1) Define a map $\Phi / X \rightarrow[\hat{0},[X]]$ by mapping the flat containing $Y$ (in the contraction $\mathcal{F} / X)$ to the flat $[Y \cup X]$ of $(E, \mathscr{F})$. The fact that this map is welldefined follows from the identity: $(\mathcal{F} / X) / Y=\mathscr{F} /(X \cup Y)$ for $X \in \mathcal{F}$ and $Y \in \mathcal{F} / X$. 
This identity also implies that the map is injective. It remains to show that the map is surjective. Let $[Z] \leq[X]$. Then $\xi(X) \subseteq \xi(Z)$. Hence, there exists $Z^{\prime}$ containing $X$ with $Z^{\prime}$ maximal among the feasible sets contained in $\xi(Z)$. Therefore, $Z^{\prime} \backslash X \in$ $\mathcal{F} / X$, and $Z^{\prime} \backslash X$ maps to the flat containing $\left(Z^{\prime} \backslash X\right) \cup X=Z^{\prime}$, which is [Z] by Proposition 2.4.

(2) Suppose $x \in(\Gamma / X)(Y)$. Then $Y \cup x \in \mathcal{F} / X$. So $(X \cup Y) \cup x \in \mathcal{F}$. That is, $x \in \Gamma(X \cup Y)$. Conversely, suppose $x \in \Gamma(X \cup Y)$. Then $X \cup(Y \cup x) \in \mathcal{F}$, and so $(Y \cup x) \in \mathcal{F} / X$. That is, $x \in(\Gamma / X)(Y)$.

(3) Let $x \in(\xi / X)(Y)$. Then $x \in W$ for some $W \in \mathcal{F} / X$ that is equivalent (in $\mathcal{F} / X)$ to $Y$. So $x \in \bigcup_{Z \in \mathcal{F} / X} Z$. And since the map defined in (1) is well-defined, we have $[X \cup Y]=[X \cup W]$. Hence, $x \in W \subseteq \xi(X \cup W)=\xi(X \cup Y)$.

We remark that the containment in the previous result can be proper.

\subsubsection{Contractions of Oriented Interval Greedoids}

Let $(E, \mathcal{F}, \mathcal{G})$ be an oriented interval greedoid and $\Phi=\operatorname{supp}(\mathcal{G})$ the lattice of flats of $(E, \mathcal{F})$. Recall that $\mathcal{G}$ with the operation $\circ$ forms a semigroup (Proposition 3.4 and (OG3)). For $A \in \Phi$, let

$$
\mathcal{G}_{\leq A}=\{\alpha \in \mathcal{G}: \operatorname{supp}(\alpha) \leq A\}
$$

Then $\mathcal{G}_{\leq A}$ is a subsemigroup of $\mathcal{G}$. We will show that it is isomorphic to an oriented interval greedoid over the contraction of $(E, \mathcal{F})$ by $X \in \mathcal{F}$, where $A=[X]$.

Let $\alpha$ be a covector of $(E, \mathcal{F})$ with $\operatorname{supp}(\alpha) \leq[X]$. By definition of the partial order, there exists $Y \in \mathcal{F} / X$ such that $\operatorname{supp}(\alpha)=[X \cup Y]$. Therefore, $Y$ is a feasible set in the contracted interval greedoid and so it makes sense to talk about its flat $(\Phi / X)(Y)$. By restricting $\alpha$ to the subset $(\Gamma / X)(Y)$, we get a signed flat $\left((\Phi / X)(Y),\left.\alpha\right|_{(\Gamma / X)(Y)}\right)$ of the contracted interval greedoid. We denote the covector of this signed flat by $\operatorname{con}_{X}(\alpha)$. Then,

$$
\operatorname{con}_{X}(\alpha)(e)= \begin{cases}0, & \text { if } e \in(\xi / X)(Y), \\ \alpha(e), & \text { if } e \in(\Gamma / X)(Y), \\ 1, & \text { otherwise. }\end{cases}
$$

It follows from Proposition 4.1 that if $\operatorname{con}_{X}(\alpha)(e) \neq 1$, then $\operatorname{con}_{X}(\alpha)(e)=\alpha(e)$.

Lemma 4.2 Suppose $(E, \mathcal{F})$ is an interval greedoid and let $X \in \mathcal{F}$. Let $\alpha$ and $\beta$ be covectors of $(E, \mathcal{F})$ with $\operatorname{supp}(\alpha), \operatorname{supp}(\beta) \leq[X]$.

(1) $(\operatorname{supp} / X)\left(\operatorname{con}_{X}(\alpha)\right)=(\Phi / X)(Y)$ and $(\operatorname{supp} / X)\left(\operatorname{con}_{X}(\beta)\right)=(\Phi / X)(Z)$, where $Y, Z \in \mathcal{F} / X$ satisfy $[X \cup Y]=\operatorname{supp}(\alpha)$ and $[X \cup Z]=\operatorname{supp}(\beta)$.

(2) $\operatorname{con}_{X}(\alpha) \circ \operatorname{con}_{X}(\beta)=\operatorname{con}_{X}(\alpha \circ \beta)$.

Proof (1) Since $\operatorname{con}_{X}(\alpha)$ is the covector of the signed flat $\left((\Phi / X)(Y),\left.\alpha\right|_{(\Gamma / X)(Y)}\right)$, it follows from the definition of supp $/ X$ that $(\operatorname{supp} / X)\left(\operatorname{con}_{X}(\alpha)\right)=(\Phi / X)(Y)$. 
(2) We first argue that the supports of the two elements are the same. It follows from the definition of $\circ$ that the support of $\operatorname{con}_{X}(\alpha) \circ \operatorname{con}_{X}(\beta)$ is the join of their supports, so it is $(\Phi / X)(Y) \vee(\Phi / X)(Z)$ by (1). Under the isomorphism $\Phi / X \cong$ $[\hat{0},[X]]$, this corresponds to $[X \cup Y] \vee[X \cup Z]$, which we can express as $[X \cup W]$ for some $W \in \mathcal{F} / X$. Hence, $(\Phi / X)(Y) \vee(\Phi / X)(Z)=(\Phi / X)(W)$. Note that $[X \cup W]$ is also the support of $\alpha \circ \beta$, so (1) implies that $(\operatorname{supp} / X)\left(\operatorname{con}_{X}(\alpha \circ \beta)\right)=(\Phi / X)(W)$.

Since $\operatorname{con}_{X}(\alpha) \circ \operatorname{con}_{X}(\beta)$ and $\operatorname{con}_{X}(\alpha \circ \beta)$ are covectors of support $(\Phi / X)(W)$, to show that they are equal it suffices to show that they agree on $(\Gamma / X)(W)$. Let $e \in(\Gamma / X)(W)$. Then,

$$
\left(\operatorname{con}_{X}(\alpha) \circ \operatorname{con}_{X}(\beta)\right)(e)= \begin{cases}\operatorname{con}_{X}(\beta)(e), & \text { if } \operatorname{con}_{X}(\beta)(e)>\operatorname{con}_{X}(\alpha)(e), \\ \operatorname{con}_{X}(\alpha)(e), & \text { otherwise }\end{cases}
$$

Since $\left(\operatorname{con}_{X}(\alpha) \circ \operatorname{con}_{X}(\beta)\right)(e) \neq 1$, it follows that neither $\operatorname{con}_{X}(\alpha)(e)$ nor $\operatorname{con}_{X}(\beta)(e)$ is 1 . Hence, $\operatorname{con}_{X}(\alpha)(e)=\alpha(e)$ and $\operatorname{con}_{X}(\beta)(e)=\beta(e)$ (see the sentence following (4.1)). Therefore,

$$
\left(\operatorname{con}_{X}(\alpha) \circ \operatorname{con}_{X}(\beta)\right)(e)= \begin{cases}\beta(e), & \text { if } \beta(e)>\alpha(e), \\ \alpha(e), & \text { otherwise }\end{cases}
$$

This is precisely $(\alpha \circ \beta)(e)$, which is $\operatorname{con}_{X}(\alpha \circ \beta)(e)$ by (4.1).

Proposition 4.3 Let $(E, \mathcal{F}, \mathcal{G})$ denote an oriented interval greedoid and let $X \in \mathcal{F}$. Then

$$
\mathcal{G} / X=\left\{\operatorname{con}_{X}(\alpha): \alpha \in \mathcal{G} \text { and } \operatorname{supp}(\alpha) \leq[X]\right\}
$$

defines an oriented interval greedoid over the contraction of $(E, \mathcal{F})$ by $X$.

Proof (OG1). Let $A \in \Phi / X$. Then $A=(\Phi / X)(Y)$ for some $Y \in \mathcal{F} / X$, and so $[Y \cup$ $X] \in \Phi$. Since $\mathcal{G}$ satisfies (OG1), there exists $\alpha \in \mathcal{G}$ with $\operatorname{supp}(\alpha)=[Y \cup X] \leq[X]$. Then $\operatorname{con}_{X}(\alpha) \in \mathcal{G} / X$ and $(\operatorname{supp} / X)\left(\operatorname{con}_{X}(\alpha)\right)=(\Phi / X)(Y)=A$ by Lemma 4.2.

(OG2) Suppose $v \in \mathcal{G} / X$. Then there exists some $\beta \in \mathcal{G}$ such that $\operatorname{supp}(\beta) \leq[X]$ and $\operatorname{con}_{X}(\beta)=v$. Then $-\beta \in \mathcal{G}$ by (OG2), and so $-v=\operatorname{con}_{X}(-\beta) \in \mathcal{G} / X$.

(OG3) Suppose $\operatorname{con}_{X}(\alpha)$ and $\operatorname{con}_{X}(\beta)$ are in $\mathcal{G} / X$. Then $\alpha \circ \beta \in \mathcal{G}$, by (OG3), and $\operatorname{supp}(\alpha \circ \beta)=\operatorname{supp}(\alpha) \vee \operatorname{supp}(\beta) \leq[X]$. Therefore, $\operatorname{con}_{X}(\alpha \circ \beta) \in \mathcal{G} / X$. By Lemma 4.2, $\operatorname{con}_{X}(\alpha \circ \beta)=\operatorname{con}_{X}(\alpha) \circ \operatorname{con}_{X}(\beta)$, so $\operatorname{con}_{X}(\alpha) \circ \operatorname{con}_{X}(\beta) \in \mathcal{G} / X$.

(OG4) Suppose $\operatorname{con}_{X}(\alpha), \operatorname{con}_{X}(\beta) \in \mathcal{G} / X$, and let $x \in(S / X)\left(\operatorname{con}_{X}(\alpha), \operatorname{con}_{X}(\beta)\right)$ such that $\left(\operatorname{con}_{X}(\alpha) \circ \operatorname{con}_{X}(\beta)\right)(x) \neq 1$.

Since $\operatorname{con}_{X}(\alpha)(x)=-\operatorname{con}_{X}(\beta)(x) \in\{+,-\}$, it follows from (4.1) that $\alpha(x)=$ $-\beta(x) \in\{+,-\}$. Hence, $x \in S(\alpha, \beta)$. Since $\operatorname{con}_{X}(\alpha \circ \beta)=\operatorname{con}_{X}(\alpha) \circ \operatorname{con}_{X}(\beta)$, it follows that $\operatorname{con}_{X}(\alpha \circ \beta)(x) \neq 1$, which implies that $(\alpha \circ \beta)(x) \neq 1$. Therefore, (OG4) applies to $\alpha, \beta$ and $x$ to guarantee the existence of $\gamma \in \mathcal{G}$ satisfying $\gamma(x)=0$ and for all $y \notin S(\alpha, \beta)$, if $(\alpha \circ \beta)(y) \neq 1$, then $\gamma(y)=(\alpha \circ \beta)(y)=(\beta \circ \alpha)(y)$. We claim that $\operatorname{con}_{X}(\gamma)$ satisfies the conditions of (OG4) for $\mathcal{G} / X$.

We first show that $\operatorname{supp}(\gamma) \leq \operatorname{supp}(\alpha \circ \beta)$. Indeed, if $(\alpha \circ \beta)(y)=0$, then $\alpha(y)=0$ and $\beta(y)=0$, so $y \notin S(\alpha, \beta)$. We conclude from (OG4) that $\gamma(y)=(\alpha \circ \beta)(y)=0$. 
Next we argue that $\operatorname{con}_{X}(\gamma)(x)=0$. Since $\operatorname{supp}(\gamma) \leq \operatorname{supp}(\alpha \circ \beta)$, it follows that $(\operatorname{supp} / X)\left(\operatorname{con}_{X}(\gamma)\right) \leq(\operatorname{supp} / X)\left(\operatorname{con}_{X}(\alpha \circ \beta)\right)$. Then Proposition 3.3 and the assumption that $\operatorname{con}_{X}(\alpha \circ \beta)(x) \neq 1$ implies that $\operatorname{con}_{X}(\gamma)(x) \neq 1$. If $\operatorname{con}_{X}(\gamma)(x) \in\{+,-\}$, then $\gamma(x) \in\{+,-\}$ contradicting the fact that $\gamma(x)=0$. Therefore, $\operatorname{con}_{X}(\gamma)(x)=0$.

Now let $y \in \bigcup_{Z \in \mathcal{F} / X} Z$ with $y \notin(S / X)\left(\operatorname{con}_{X}(\alpha), \operatorname{con}_{X}(\beta)\right)$. We claim that $y \notin$ $S(\alpha, \beta)$. If $y \in S(\alpha, \beta)$, then $\alpha(y)=-\beta(y) \in\{+,-\}$, and so $\operatorname{con}_{X}(\alpha)(y)=\alpha(y)=$ $-\beta(y)=-\operatorname{con}_{X}(\beta)(y) \in\{+,-\}$ by (4.1), a contradiction.

Now suppose that $\operatorname{con}_{X}(\alpha \circ \beta)(y) \neq 1$. As above, Proposition 3.3 implies that $\operatorname{con}_{X}(\gamma)(y) \neq 1$. Then the sentence following (4.1) implies that $\operatorname{con}_{X}(\alpha \circ \beta)(y)=$ $(\alpha \circ \beta)(y)$ and that $\operatorname{con}_{X}(\gamma)(y)=\gamma(y)$. Hence, $(\alpha \circ \beta)(y) \neq 1$, so $\gamma(y)=(\alpha \circ$ $\beta)(y)=(\beta \circ \alpha)(y)$ by (OG4). Therefore, $\operatorname{con}_{X}(\gamma)(y)=\left(\operatorname{con}_{X}(\alpha) \circ \operatorname{con}_{X}(\beta)\right)(y)=$ $\left(\operatorname{con}_{X}(\beta) \circ \operatorname{con}_{X}(\alpha)\right)(y)$.

The following result identifies $\mathcal{G} / X$ with a subsemigroup of $\mathcal{G}$.

Proposition 4.4 Let $(E, \mathcal{F}, \mathcal{G})$ denote an oriented interval greedoid and let $X \in \mathcal{F}$. Then there is a semigroup isomorphism

$$
\mathcal{G}_{\leq[X]} \cong \mathcal{G} / X
$$

given by mapping $\alpha \in \mathcal{G}$ with $\operatorname{supp}(\alpha) \leq[X]$ to $\operatorname{con}_{X}(\alpha)$.

Proof Lemma 4.2 shows this is a semigroup morphism. The morphism is surjective by definition of $\mathcal{G} / X$. It remains to show that the morphism is injective. Suppose $\operatorname{con}_{X}(\alpha)=\operatorname{con}_{X}(\beta)$. Then $\operatorname{supp}(\alpha)=\operatorname{supp}(\beta)$, which can be written as $[X \cup Y]$. Now $(\Gamma / X)(Y)=\Gamma(X \cup Y)$, so $\alpha$ and $\beta$ agree on $\Gamma(X \cup Y)$, and they each are zero on exactly $\xi([X \cup Y])$, so $\alpha$ and $\beta$ agree, as desired.

\subsection{Restriction}

We introduce a restriction operation for an oriented interval greedoid $(E, \mathcal{F}, \mathcal{G})$ that produces an oriented interval greedoid on a restriction of the interval greedoid $(E, \mathcal{F})$. We begin by recalling restriction for interval greedoids.

\subsubsection{Restriction of an Interval Greedoid}

Let $(E, \mathcal{F})$ denote an interval greedoid and $\Phi$ its lattice of flats. If $W \subseteq E$ is an arbitrary subset, then the restriction of $(E, \mathcal{F})$ to $W$ is the interval greedoid $\left(W,\left.\mathcal{F}\right|_{W}\right)$, where

$$
\left.\mathcal{F}\right|_{W}=\{X \in \mathcal{F}: X \subseteq W\}
$$

To distinguish between objects defined for $(E, \mathcal{F})$ and $\left(W,\left.\mathcal{F}\right|_{W}\right)$, we take the following convention. If $\Xi$ is an object defined for $(E, \mathcal{F}$ ) (for example, its lattice of flats $\Phi$, the set of continuations $\Gamma$ ), then $\left.\Xi\right|_{W}$ will denote the corresponding object defined for $\left(W,\left.\mathcal{F}\right|_{W}\right)$ (for example, $\left.\left.\Phi\right|_{W},\left.\Gamma\right|_{W}\right)$. 
There is a map $\left.\Phi \rightarrow \Phi\right|_{W}$ that maps a flat $C \in \Phi$ onto the flat $\left.\mu\right|_{W}(W \cap \xi(C))$. We denote the image of $C$ by $\left.C\right|_{W}$. Note that if $\left.Y \in \mathcal{F}\right|_{W}$, then $Y \in \mathcal{F}$ and the image of $[Y] \in \Phi$ under $\left.\Phi \rightarrow \Phi\right|_{W}$ is the flat in $\left.\Phi\right|_{W}$ that contains $Y$, which by our above convention is denoted by $\left.[Y]\right|_{W}$.

Lemma 4.5 Suppose $(E, \mathcal{F})$ is an interval greedoid and let $W \subseteq E$.

(1) If $\left.Y \in \mathcal{F}\right|_{W}$, then $\left.\Gamma\right|_{W}(Y)=W \cap \Gamma(Y)$.

(2) If $\left.Y \in \mathcal{F}\right|_{W}$ and $\xi(Y) \subseteq W$, then $\left.\xi\right|_{W}(Y)=\xi(Y)$.

(3) If $A \in \Phi$, then $\left.\Gamma\right|_{W}\left(\left.A\right|_{W}\right) \subseteq W \cap \Gamma(A)$.

(3) If $A \in \Phi$, then $\left.\xi\right|_{W}\left(\left.A\right|_{W}\right) \subseteq W \cap \xi(A)$.

Proof (1) If $\left.Y \in \mathcal{F}\right|_{W}$, then $\Gamma_{W}(Y)=\{y \in W \backslash Y: Y \cup y \in \mathcal{F}\}=W \cap\{y \in E \backslash Y$ : $Y \cup y \in \mathcal{F}\}=W \cap \Gamma(Y)$.

(2) Suppose $\left.Y \in \mathcal{F}\right|_{W}$ and $\xi(Y) \subseteq W$. The latter assumption implies that all feasible sets that are equivalent to $Y$ in $(E, \mathcal{F})$ are contained in $W$. So they are contained in $\left.\mathcal{F}\right|_{W}$. Moreover, they are also equivalent in $\left.\mathcal{F}\right|_{W}$ since they are all maximal among the feasible sets contained in $\xi(Y)$ (see Proposition 2.4).

(3) Let $\left.Z \in A\right|_{W}$ and let $\left.x \in \Gamma\right|_{W}\left(\left.A\right|_{W}\right)=\left.\Gamma\right|_{W}(Z)$. By (1), $x \in W \cap \Gamma(Z)$. Since $\left.A\right|_{W}=\left.\mu\right|_{W}(W \cap \xi(A))$, there exists $Y \in \mathcal{F}$ containing $Z$ that is maximal among the feasible sets contained in $\xi(A)$. Thus, $[Z] \geq[Y]=A$, and by Proposition 3.3,

$$
\left.\Gamma\right|_{W}(Z)=(\Gamma(Z) \cap W) \subseteq(\Gamma(A) \cap W) \cup(\xi(A) \cap W) .
$$

If $x \in W \cap \xi(A)$, then $Z \cup x \in W \cap \xi(A)$, contradicting that $Z$ is maximal among the feasible sets contained in $W \cap \xi(A)$. Therefore, $x \in W \cap \Gamma(A)$.

(4) By definition $\left.A\right|_{W}=\left.\mu\right|_{W}(W \cap \xi(A))$, so the sets contained in $\left.A\right|_{W}$ are the sets that are maximal among the feasible sets contained in $W \cap \xi(A)$. Let $D$ be a maximal feasible set in $W \cap \xi(A)$, and let $C$ be a set in $W$ that is equivalent to $D$ in the restriction. We want to show that $C$ is contained in $\xi(A)$.

Since $C$ and $D$ are equivalent in the restriction, they have the same continuations inside $W$. Let $x$ be a continuation of $C$ with $x \notin W$. Then $D$ can be augmented from $C \cup x$, and clearly $D$ cannot be augmented from $C$, so it can be augmented by $x$. Thus $\Gamma(C)$ contains $\Gamma(D)$, and the converse is also true. So $C$ and $D$ have the same continuations in the original interval greedoid, and therefore are equivalent. In particular, $C$ is in $\xi(A)$ as well.

Remark 4.6 The inclusions in (3) and (4) can be proper, as can be seen in the following example. Let $E=\{a, b, c\}$ and $\mathcal{F}=\{\varnothing,\{a\},\{a, b\},\{a, c\}\}$. Then $(E, \mathcal{F})$ is an interval greedoid. If $W=\{b, c\}$, then $\left.\mathcal{F}\right|_{W}=\{\varnothing\}$, so

$$
\begin{gathered}
\left.\Gamma\right|_{W}\left(\left.[\{a\}]\right|_{W}\right)=\left.\Gamma\right|_{W}(\varnothing)=\varnothing \subsetneq \Gamma([\{a\}]) \cap W=\{b, c\}, \\
\left.\xi\right|_{W}\left(\left.[\{a, b\}]\right|_{W}\right)=\left.\xi\right|_{W}(\varnothing)=\varnothing \subsetneq \xi([\{a, b\}]) \cap W=\{b, c\} .
\end{gathered}
$$

Proposition 4.7 The map $\left.\Phi \rightarrow \Phi\right|_{W}$ defined by $\left.C \mapsto C\right|_{W}=\left.\mu\right|_{W}(W \cap \xi(C))$ for all $C \in \Phi$ is order-preserving, surjective and preserves joins: $\left.(A \vee B)\right|_{W}=\left.\left.A\right|_{W} \vee B\right|_{W}$ for all $A, B \in \Phi$. 
Proof The mapping is order-preserving since $\xi$ and $\left.\mu\right|_{W}$ are order-reversing. The map is surjective since if $\left.\left.[Y]\right|_{W} \in \Phi\right|_{W}$, then it follows that $Y \in \mathcal{F}$ and that $Y$ is maximal among the feasible subsets contained in $W \cap \xi([Y])$. So, $\left.[Y] \mapsto[Y]\right|_{W}$ under this mapping.

Since $A, B \leq A \vee B$, and since the map is order-preserving, $\left.\left.A\right|_{W} \vee B\right|_{W} \leq$ $\left.(A \vee B)\right|_{W}$. Since $\left.\left.\left.A\right|_{W} \vee B\right|_{W} \in \Phi\right|_{W}$, there exists $\left.Z \in \mathcal{F}\right|_{W}$ such that $\left.\left.A\right|_{W} \vee B\right|_{W}=$ $\left.[Z]\right|_{W}$. Then $Z$ is maximal among the feasible sets contained in $\left.\xi\right|_{W}\left(\left.A\right|_{W}\right) \cap$ $\left.\xi\right|_{W}\left(\left.B\right|_{W}\right)$ by definition of $\vee$ (Proposition 2.9). Since $\left.A\right|_{W}$ is the collection of sets that are maximal among the feasible sets contained in $W \cap \xi(A)$, it follows that $\left.\xi\right|_{W}\left(\left.A\right|_{W}\right) \subseteq W \cap \xi(A)$. Therefore, $\left.\left.\xi\right|_{W}\left(\left.A\right|_{W}\right) \cap \xi\right|_{W}\left(\left.B\right|_{W}\right) \subseteq W \cap \xi(A) \cap \xi(B)$. So there exists $Y$ containing $Z$ that is maximal among the feasible sets contained in $W \cap \xi(A) \cap \xi(B)$. We now argue that $Y$ is maximal among the feasible sets contained in $W \cap \xi(A \vee B)$. There exists $U$ containing $Y$ that is maximal among the feasible sets contained in $\xi(A) \cap \xi(B)$. Thus, $[Y] \geq[U]=A \vee B$. This implies $Y \subseteq \xi(Y) \subseteq \xi(A \vee B)$ since $\xi$ is order-reversing. So $Y \subseteq W \cap \xi(A \vee B)$. Since $\xi(A \vee B) \subseteq \xi(A) \cap \xi(B)$, it follows that $Y$ is maximal among the feasible sets contained in $W \cap \xi(A \vee B)$. Therefore, $\left.(A \vee B)\right|_{W}=\left.[Y]\right|_{W}$. Since $Y \supseteq Z$, we have $\left.[Y]\right|_{W} \leq\left.[Z]\right|_{W}$. Thus, $\left.(A \vee B)\right|_{W} \leq\left.\left. A\right|_{W} \vee B\right|_{W}$. Therefore, $\left.(A \vee B)\right|_{W}=$ $\left.\left.A\right|_{W} \vee B\right|_{W}$.

Since $\left.\mathcal{F}\right|_{W} \subseteq \mathcal{F}$, there is also a map in the reverse direction $\left.\Phi\right|_{W} \rightarrow \Phi$, defined by $A \mapsto[Y]$ for any $Y \in A$. Proposition 2.4 implies the map is well-defined, and the identity $\left(\left.\mathcal{F}\right|_{W}\right) / Y=\left.(\mathcal{F} / Y)\right|_{W}=\{X \subseteq W \backslash Y: X \cup Y \in \mathcal{F}\}$ implies the map is injective. It is order-preserving and its image is contained in the interval $[[X], \hat{1}]$, where $X$ is maximal among the feasible sets contained in $W$.

Unlike for matroids, for an arbitrary interval greedoid, the lattice of flats $\left.\Phi\right|_{W}$ is not, in general, an interval of $\Phi$. However, if $W \supseteq \xi(X)$, where $X$ is maximal among the feasible subsets contained in $W$, then $\left.\Phi\right|_{W} \cong[[X], \hat{1}] \subseteq \Phi$. (This is obtained by considering the compositions of the maps $\left.\Phi\right|_{W} \rightarrow \Phi$ and $\left.\Phi \rightarrow \Phi\right|_{W}$ defined above.)

\subsubsection{Restricting Covectors}

Let $W \subseteq E$ and let $\alpha$ be a covector of $(E, \mathcal{F})$. Let $A=\operatorname{supp}(\alpha)$. It follows from Lemma 4.5 that $\left.\Gamma\right|_{W}\left(\left.A\right|_{W}\right) \subseteq \Gamma(A)$, so $\left(\left.A\right|_{W},\left.\alpha\right|_{\left.\left.\Gamma\right|_{W}\left(\left.A\right|_{W}\right)\right)}\right)$ is a signed flat of $\left(W,\left.\mathcal{F}\right|_{W}\right)$. Let $\operatorname{res}_{W}(\alpha)$ denote the covector of this signed flat:

$$
\operatorname{res}_{W}(\alpha)(w)= \begin{cases}0, & \text { if }\left.w \in \xi\right|_{W}\left(\left.A\right|_{W}\right), \\ \alpha(w), & \text { if }\left.w \in \Gamma\right|_{W}\left(\left.A\right|_{W}\right) \\ 1, & \text { otherwise }\end{cases}
$$

for all $w \in W$. Observe that by construction $\left.\operatorname{supp}\right|_{W}\left(\operatorname{res}_{W}(\alpha)\right)=\left.\operatorname{supp}(\alpha)\right|_{W}$. Also note that by Lemma 4.5, if $\operatorname{res}_{W}(\alpha)(w) \neq \alpha(w)$, then $\operatorname{res}_{W}(\alpha)(w)=1$.

Example 4.8 (Antimatroid from three colinear points) Let $(E, \mathcal{F}, \mathcal{G})$ be the oriented interval greedoid arising from the convex geometry of three colinear points, $x, y, z$ with $y$ between $x, z$. Let $W=\{x, y\}$. The covectors of $\left.\mathcal{G}\right|_{W}$ are: $( \pm, 1) ;(0, \pm) ;(0,0)$. 
For $\alpha \in \mathcal{G}$, if $\alpha(z) \neq 0$, then $\operatorname{res}_{W}(\alpha)$ equals the restriction of $\alpha$ to $W$. However, if, for example, $\alpha=(+,+, 0)$, then $\operatorname{res}_{W}(\alpha)=(+, 1)$.

As we have just seen in an example, $\operatorname{res}_{W}(\alpha)$ cannot necessarily be obtained by restricting $\alpha$ to $W$. The following proposition sheds more light on this.

Proposition 4.9 Suppose $(E, \mathcal{F})$ is an interval greedoid and let $W \subseteq E$.

(1) If $\alpha$ is a covector of $(E, \mathcal{F})$ and $A=\operatorname{supp}(\alpha)$, then $\operatorname{res}_{W}(\alpha)=\left.\alpha\right|_{W}$ if and only if $\left.\xi\right|_{W}\left(\left.A\right|_{W}\right)=W \cap \xi(A)$ and $\left.\Gamma\right|_{W}\left(\left.A\right|_{W}\right)=W \cap \Gamma(A)$.

(2) If $\alpha$ and $\beta$ are covectors of $(E, \mathcal{F})$, then $\operatorname{res}_{W}(\alpha \circ \beta)=\operatorname{res}_{W}(\alpha) \circ \operatorname{res}_{W}(\beta)$.

Proof (1) This is obvious from the definitions.

(2) Let $A=\operatorname{supp}(\alpha)$ and $B=\operatorname{supp}(\beta)$. By Sect. 4.2.1, $\left.\left.A\right|_{W} \vee B\right|_{W}=\left.(A \vee B)\right|_{W}$. Thus, $\operatorname{res}_{W}(\alpha) \circ \operatorname{res}_{W}(\beta)$ and $\operatorname{res}_{W}(\alpha \circ \beta)$ have the same support. It is therefore clear that they coincide.

\subsubsection{Restriction of an Oriented Interval Greedoid}

The following results shows that the covectors obtained by restricting the covectors of an oriented interval greedoid $(E, \mathcal{F}, \mathcal{G})$ satisfy the first three axioms for an oriented interval greedoid.

Proposition 4.10 Suppose $(E, \mathcal{F}, \mathcal{G})$ is an oriented interval greedoid. If $W \subseteq E$, then $\left(W,\left.\mathcal{F}\right|_{W},\left.\mathcal{G}\right|_{W}\right)$ satisfies $(O G 1),(O G 2)$ and $(O G 3)$, where

$$
\left.\mathcal{G}\right|_{W}=\left\{\operatorname{res}_{W}(\alpha): \alpha \in \mathcal{G}\right\} .
$$

Proof By construction, we have that $\left.\mathcal{G}\right|_{W}$ is a collection of covectors of $\left(W,\left.\mathcal{F}\right|_{W}\right)$ and that $\operatorname{supp}_{W}\left(\operatorname{res}_{W}(\alpha)\right)=\left.\left.\operatorname{supp}(\alpha)\right|_{W} \in \Phi\right|_{W}$.

(OG1) Let $\left.A \in \Phi\right|_{W}$ and let $Y \in A$. Then $[Y] \in \Phi$ and so there exists $\alpha \in \mathcal{G}$ with $\operatorname{supp}(\alpha)=[Y]$. So $\left.\operatorname{res}_{W}(\alpha) \in \mathcal{G}\right|_{W}$ and $\left.\operatorname{supp}\right|_{W}\left(\operatorname{res}_{W}(\alpha)\right)=\left.\operatorname{supp}(\alpha)\right|_{W}=\left.A\right|_{W}$.

(OG2) If $\left.\operatorname{res}_{W}(\alpha) \in \mathcal{G}\right|_{W}$, then $-\alpha \in \mathcal{G}$. Hence, $-\operatorname{res}_{W}(\alpha)=\left.\operatorname{res}_{W}(-\alpha) \in \mathcal{G}\right|_{W}$.

(OG3) Suppose $\operatorname{res}_{W}(\alpha),\left.\operatorname{res}_{W}(\beta) \in \mathcal{G}\right|_{W}$ and let $A=\operatorname{supp}(\alpha)$ and $B=\operatorname{supp}(\beta)$. Then $\alpha \circ \beta \in \mathcal{G}$, and so $\left.\operatorname{res}_{W}(\alpha \circ \beta) \in \mathcal{G}\right|_{W}$. By Proposition 4.9, $\operatorname{res}_{W}(\alpha \circ \beta)=$ $\operatorname{res}_{W}(\alpha) \circ \operatorname{res}_{W}(\beta)$, so $\left.\operatorname{res}_{W}(\alpha) \circ \operatorname{res}_{W}(\beta) \in \mathcal{G}\right|_{W}$.

Although (OG4) may not hold for an arbitrary restriction, it does hold for certain restrictions, so we get an oriented interval greedoid.

Theorem 4.11 Suppose $(E, \mathcal{F}, \mathcal{G})$ is an oriented interval greedoid and let $W \subseteq E$. If $\operatorname{res}_{W}(\alpha)=\left.\alpha\right|_{W}$ for all $\alpha \in \mathcal{G}$, then $\left(W,\left.\mathcal{F}\right|_{W},\left.\mathcal{G}\right|_{W}\right)$ is an oriented interval greedoid.

Proof (OG1)-(OG3) hold by Proposition 4.10. The assumption that $\operatorname{res}_{W}(\alpha)=\left.\alpha\right|_{W}$ for all $\alpha \in \mathcal{G}$ means that (OG4) for $\mathcal{G}$ implies (OG4) for $\left(W,\left.\mathcal{F}\right|_{W},\left.\mathcal{G}\right|_{W}\right)$.

Sections 4.3 and 4.4 describe restriction to two particular types of subset of $E$. 


\subsection{Restriction to $\Gamma(\varnothing)$}

In this section we treat restriction to $\Gamma(\varnothing)$.

Proposition 4.12 Suppose $(E, \mathcal{F}, \mathcal{G})$ is an oriented interval greedoid. Then the restriction $\left(\Gamma(\varnothing),\left.\mathcal{F}\right|_{\Gamma(\varnothing)},\left.\mathcal{G}\right|_{\Gamma(\varnothing)}\right)$ is an oriented matroid, and

$$
\left.\mathcal{G}\right|_{\Gamma(\varnothing)}=\left\{\left.\alpha\right|_{\Gamma(\varnothing)}: \alpha \in \mathcal{G}\right\} .
$$

Proof Since $\left(\Gamma(\varnothing),\left.\mathcal{F}\right|_{\Gamma(\varnothing)}\right)$ is a matroid, it will follow from Theorem 3.12 that $\left(\Gamma(\varnothing),\left.\mathcal{F}\right|_{\Gamma(\varnothing)},\left.\mathcal{G}\right|_{\Gamma(\varnothing)}\right)$ is an oriented matroid once we show that it is oriented interval greedoid. By Proposition 4.9 and Theorem 4.11, we need only show that $\left.\Gamma\right|_{\Gamma(\varnothing)}\left(\left.A\right|_{\Gamma(\varnothing)}\right)=\Gamma(\varnothing) \cap \Gamma(A)$ and $\left.\xi\right|_{\Gamma(\varnothing)}\left(\left.A\right|_{\Gamma(\varnothing)}\right)=\Gamma(\varnothing) \cap \xi(A)$.

Suppose $x \in \Gamma(\varnothing) \cap \Gamma(A)$. Let $\left.Y \in A\right|_{\Gamma(\varnothing)}$. Then $Y \subseteq \Gamma(\varnothing) \cap \xi(A)$, so there exists $Z \supseteq Y$ such that $Z$ is maximal among the sets in $\mathscr{F}$ contained in $\xi(A)$. By Proposition 2.4, $Z \in A$, so $\Gamma(A)=\Gamma(Z)$. Hence, $Z \cup x \in \mathcal{F}$ since $x \in \Gamma(A)$. Also, $\{x\} \in \mathcal{F}$ since $x \in \Gamma(\varnothing)$. Therefore, (IG3) applied to $\varnothing \subseteq Y \subseteq Z$ implies $Y \cup$ $x \in \mathcal{F}$. Since $Y \cup x \subseteq \Gamma(\varnothing)$, we have $\left.Y \cup x \in \mathcal{F}\right|_{\Gamma(\varnothing)}$. So $\left.x \in \Gamma\right|_{\Gamma(\varnothing)}\left(\left.A\right|_{\Gamma(\varnothing)}\right)$. This establishes one inclusion. The reverse inclusion follows from Lemma 4.5.

It remains to show that $\left.\xi\right|_{\Gamma(\varnothing)}\left(\left.A\right|_{\Gamma(\varnothing)}\right)=\Gamma(\varnothing) \cap \xi(A)$. Suppose $x \in \Gamma(\varnothing) \cap$ $\xi(A)$. Then $\{x\} \in \mathcal{F}$ since $x \in \Gamma(\varnothing)$. Therefore, there exists $Y$ containing $x$ such that $Y$ is maximal among the feasible sets contained in $\Gamma(\varnothing) \cap \xi(A)$. Then $\left.Y \in A\right|_{\Gamma(\varnothing)}$. Therefore, $\left.Y \subseteq \xi\right|_{\Gamma(\varnothing)}\left(\left.A\right|_{\Gamma(\varnothing)}\right)$, and so $\left.x \in \xi\right|_{\Gamma(\varnothing)}\left(\left.A\right|_{\Gamma(\varnothing)}\right)$. This, combined with Lemma 4.5, establishes the equality $\left.\xi\right|_{\Gamma(\varnothing)}\left(\left.A\right|_{\Gamma(\varnothing)}\right)=\Gamma(\varnothing) \cap \xi(A)$.

\subsection{Restriction to $\xi(X)$}

To simplify notation, we write $\xi(X)$ for $\xi([X])$ for any feasible set $X \in \mathcal{F}$.

We show that restriction to $\xi(X)$ for $X \in \mathcal{F}$ produces an oriented interval greedoid $\left(\xi(X),\left.\mathcal{F}\right|_{\xi(X)},\left.\mathcal{G}\right|_{\xi(X)}\right)$ and that there is a semigroup isomorphism

$$
\left.\mathcal{G}\right|_{\xi(X)} \stackrel{\cong}{\longrightarrow} \mathcal{G}_{\geq \alpha}=\{\beta \in \mathcal{G}: \beta \geq \alpha\}=\{\alpha \circ \beta: \beta \in \mathcal{G}\},
$$

where $\alpha$ is any covector with $\operatorname{supp}(\alpha)=[X]$.

Lemma 4.13 Suppose $(E, \mathcal{F})$ is an interval greedoid and let $X \in \mathcal{F}$ and $A \in \Phi$.

(1) $\left.A\right|_{\xi(X)}=A \vee[X]$

(2) $\left.\xi\right|_{\xi(X)}\left(\left.A\right|_{\xi(X)}\right)=\xi(A \vee[X])=\xi(A \vee[X]) \cap \xi(X)$.

(3) $\left.\Gamma\right|_{\xi(X)}\left(\left.A\right|_{\xi(X)}\right)=\Gamma(A \vee[X]) \cap \xi(X)$.

Proof (1) $\left.A\right|_{\xi(X)}$ is $\left.\mu\right|_{\xi(X)}(\xi(X) \cap \xi(A))$, the flat that consists of the sets that are maximal among the feasible sets contained in $\xi(X) \cap \xi(A)$. By Proposition 2.9, this is $A \vee[X]$.

(2) This follows from (1) since all feasible sets in $A \vee[X]$ are contained in $\xi(X)$.

(3) Write $A \vee[X]=[Y]$ for some $Y \in \mathcal{F}$. Then $[X] \leq[Y]$, so $\left.Y \in \mathcal{F}\right|_{\xi(X)}$ since $\xi(Y) \subseteq \xi(X)$. Thus, $\left.\Gamma\right|_{\xi(X)}\left(\left.A\right|_{\xi(X)}\right)=\left.\Gamma\right|_{\xi(X)}(Y)=\{y \in \xi(X) \backslash Y: Y \cup y \in \mathcal{F}\}=$ $\xi(X) \cap \Gamma(Y)=\xi(X) \cap \Gamma(A \vee[X])$. 
Lemma 4.14 Suppose $(E, \mathcal{F}, \mathcal{G})$ is an oriented interval greedoid and let $X \in \mathcal{F}$.

$$
\begin{aligned}
\left.\mathcal{G}\right|_{\xi(X)} & =\{\operatorname{res} \xi(X) \\
& =\left\{\left.\alpha\right|_{\xi(X)}: \alpha \in \mathcal{G} \text { and } \operatorname{supp}(\alpha) \geq[X]\right\} .
\end{aligned}
$$

Proof We begin by proving the first equality. We show that if $\beta \in \mathcal{G}$, then there exists $\alpha \in \mathcal{G}$ with $\operatorname{supp}(\alpha) \geq[X]$ and $\operatorname{res}_{\xi(X)}(\alpha)=\operatorname{res} \xi(X)(\beta)$. Let $\beta \in \mathcal{G}$ and let $B=\operatorname{supp}(\beta)$. Since $\mathcal{G}$ satisfies (OG1), there exists $\gamma \in \mathcal{G}$ such that $\operatorname{supp}(\gamma)=[X]$. Since $\operatorname{supp}(\gamma \circ \beta)=[X] \vee B$ and $\left.B\right|_{\xi(X)}=B \vee[X]=\left.(B \vee[X])\right|_{\xi(X)}$ by Lemma 4.13,

$$
\operatorname{res}_{\xi(X)}(\gamma \circ \beta)= \begin{cases}0, & \text { if }\left.x \in \xi\right|_{\xi(X)}\left(\left.B\right|_{\xi(X)}\right), \\ (\gamma \circ \beta)(x), & \text { if }\left.x \in \Gamma\right|_{\xi(X)}\left(\left.B\right|_{\xi(X)}\right), \\ 1, & \text { otherwise. }\end{cases}
$$

Therefore, $\operatorname{res} \xi(X)(\gamma \circ \beta)=\operatorname{res}_{\xi(X)}(\beta)$ if and only if $(\gamma \circ \beta)(x)=\beta(x)$ for $x \in$ $\left.\Gamma\right|_{\xi(X)}\left(\left.B\right|_{\xi(X)}\right)$. So suppose $\left.x \in \Gamma\right|_{\xi(X)}\left(\left.B\right|_{\xi(X)}\right)$. By Lemma 4.13, $x \in \xi(X) \cap \Gamma(B \vee$ $[X])$, and by Proposition 3.3, $x \in \xi(X) \cap(\Gamma(B) \cup \Gamma(X))$. This implies $x \in \Gamma(B)$ because $\xi(X) \cap \Gamma(X)=\varnothing$. Therefore, $(\gamma \circ \beta)(x)=\beta(x)$, and so res $\xi(X)(\gamma \circ \beta)(x)=$ $\beta(x)=\operatorname{res}_{\xi(X)}(\beta)(x)$.

We now prove the second equality. Let $\operatorname{res}_{\xi(X)}(\alpha)$ such that $\alpha \in \mathcal{G}$ and $A=$ $\operatorname{supp}(\alpha) \geq[X]$. By Lemma 4.13 we have $\left.A\right|_{\xi(X)}=A \vee[X]=A,\left.\xi\right|_{\xi(X)}\left(\left.A\right|_{\xi(X)}\right)=$ $\xi(X) \cap \xi(A)$ and $\left.\Gamma\right|_{\xi(X)}\left(\left.A\right|_{\xi(X)}\right)=\xi(X) \cap \Gamma(A)$. Therefore, by Proposition 4.9, $\operatorname{res}_{\xi(X)}(\alpha)=\left.\alpha\right|_{\xi(X)}$ for all $\alpha \in \mathcal{G} \operatorname{such}$ that $\operatorname{supp}(\alpha) \geq[X]$.

Theorem 4.15 Let $(E, \mathcal{F}, \mathcal{G})$ denote an oriented interval greedoid and let $X \in \mathcal{F}$. Then $\left(\xi(X),\left.\mathcal{F}\right|_{\xi(X)},\left.\mathcal{G}\right|_{\xi(X)}\right)$ is an oriented interval greedoid.

Proof By Proposition 4.10 we need only show that $\left.\mathcal{G}\right|_{\xi(X)}$ satisfies (OG4). Let $\operatorname{res}_{\xi(X)}(\alpha)$ and $\operatorname{res}_{\xi(X)}(\beta)$ be covectors in $\left.\mathcal{G}\right|_{\xi(X)}$. By Lemma 4.14, we can assume $\operatorname{supp}(\alpha) \geq[X], \operatorname{supp}(\beta) \geq[X], \operatorname{res}_{\xi(X)}(\alpha)=\left.\alpha\right|_{\xi(X)}$ and $\operatorname{res}_{\xi(X)}(\beta)=\left.\beta\right|_{\xi(X)}$.

Let $x \in S\left(\operatorname{res}_{\xi(X)}(\alpha), \operatorname{res}_{\xi(X)}(\beta)\right)$ with $\operatorname{res}_{\xi(X)}(\alpha \circ \beta)(x) \neq 1$. Then $x \in S(\alpha, \beta)$ and $(\alpha \circ \beta)(x) \neq 1$. By (OG4) applied to $\mathcal{G}$, there exists $\gamma \in \mathcal{G}$ such that $\gamma(x)=0$ and for all $y \notin S(\alpha, \beta)$, if $(\alpha \circ \beta)(y) \neq 1$, then $\gamma(y)=(\alpha \circ \beta)(y)=(\beta \circ \alpha)(y)$.

We show that $\operatorname{res}_{\xi(X)}(\gamma)$ satisfies the conditions of (OG4). Let $A=\operatorname{supp}(\alpha), B=$ $\operatorname{supp}(\beta)$ and $C=\operatorname{supp}(\gamma)$. Observe that $C \vee[X] \leq A \vee B$ : indeed, if $(\alpha \circ \beta)(e)=0$, then $\alpha(e)=0$, so $e \notin S(\alpha, \beta)$, which implies that $\gamma(e)=(\alpha \circ \beta)(e)=0$.

We first argue that $\operatorname{res}_{\xi(X)}(\gamma)(x)$ is 0 . By construction, it is either $\gamma(x)$ or 1. Suppose it is 1 . Then $\left.\left.x \notin \xi\right|_{\xi(X)}\left(\left.C\right|_{\xi(X)}\right) \cup \Gamma\right|_{\xi(X)}\left(\left.C\right|_{\xi(X)}\right)=\xi(C \vee[X]) \cup \Gamma(C \vee[X])$. By Proposition 3.3, since $C \vee[X] \leq A \vee B, x \notin \Gamma(A \vee B) \cup \xi(A \vee B)$. This implies $(\alpha \circ \beta)(x)=1$, which contradicts $(\alpha \circ \beta)(x) \neq 1$. Thus, $\operatorname{res} \xi(X)(\gamma)(x)=\gamma(x)=0$.

Let $y \notin S\left(\operatorname{res} \xi_{(X)}(\alpha), \operatorname{res}_{\xi(X)}(\beta)\right)$. Then $y \notin S(\alpha, \beta)$. Suppose $\operatorname{res} \xi(X)(\alpha \circ$ $\beta)(y) \neq 1$. Then $(\alpha \circ \beta)(y) \neq 1$. This implies, as above, that $\operatorname{res} \xi(X)(\gamma)(y) \neq 1$. Thus, $\operatorname{res}_{\xi(X)}(\gamma)(y)=\gamma(y)=(\alpha \circ \beta)(y)$. By Lemma 4.14, $\operatorname{res}_{\xi(X)}(\alpha \circ \beta)=\left.(\alpha \circ \beta)\right|_{\xi(X)}$, so $\operatorname{res}_{\xi(X)}(\gamma)(y)=\operatorname{res}_{\xi(X)}(\alpha \circ \beta)(y)=\left(\operatorname{res}_{\xi(X)}(\alpha) \circ \operatorname{res}_{\xi(X)}(\beta)\right)(y)$.

The following result identifies the semigroup $\left.\mathcal{G}\right|_{\xi(X)}$ with a subsemigroup of $\mathcal{G}$. 
Proposition 4.16 Let $(E, \mathcal{F}, \mathcal{G})$ denote an oriented interval greedoid and let $X \in \mathcal{F}$. Then $\operatorname{res}_{\xi(X)}(\beta) \mapsto \alpha \circ \beta$ defines a semigroup isomorphism

$$
\left.\mathcal{G}\right|_{\xi(X)} \stackrel{\cong}{\longrightarrow} \mathcal{G}_{\geq \alpha}=\{\beta \in \mathcal{G}: \beta \geq \alpha\}
$$

where $\alpha$ is any covector with $\operatorname{supp}(\alpha)=[X]$.

Proof Define a map $g:\left.\mathcal{G}_{\geq \alpha} \rightarrow \mathcal{G}\right|_{\xi(X)}$ by $\beta \mapsto \operatorname{res}_{\xi(X)}(\beta)$. Then $g$ is a semigroup morphism by Proposition 4.9. Define a map $f:\left.\mathcal{G}\right|_{\xi(X)} \rightarrow \mathcal{G}_{\geq \alpha}$ by $f\left(\operatorname{res}_{\xi(X)}(\beta)\right)=$ $\alpha \circ \beta$.

We argue that $f$ is well-defined. Suppose $\beta, \gamma \in \mathcal{G}$ with $B=\operatorname{supp}(\beta)$ and $C=$ $\operatorname{supp}(\gamma)$, and suppose $\operatorname{res}_{\xi(X)}(\beta)=\operatorname{res} \xi(X)(\gamma)$. Then the support of $\operatorname{res}_{\xi(X)}(\beta)=$ $\operatorname{res} \xi_{(X)}(\gamma)$ is $B \vee[X]=C \vee[X]$. This implies that $\operatorname{supp}(\alpha \circ \beta)=\operatorname{supp}(\alpha \circ \gamma)$ because $\operatorname{supp}(\alpha)=[X]$. Therefore, to show $\alpha \circ \beta=\alpha \circ \gamma$ it suffices to show that they agree on $\Gamma([X] \vee B)$. Let $x \in \Gamma([X] \vee B)$. Then $x \in \Gamma(X) \cup \xi(X)$ by Proposition 3.3. If $x \in \Gamma(X)$, then $(\alpha \circ \beta)(x)=\alpha(x)=(\alpha \circ \gamma)(x)$. So suppose $x \in \xi(X)$. Then $(\alpha \circ \beta)(x)=\beta(x)$ and $(\alpha \circ \gamma)(x)=\gamma(x)$. Moreover, $\left.x \in \Gamma\right|_{\xi(X)}\left(\left.B\right|_{\xi(X)}\right)$ and $\left.x \in \Gamma\right|_{\xi(X)}\left(\left.C\right|_{\xi(X)}\right)$ by Lemma 4.13. So $\operatorname{res}_{\xi(X)}(\beta)(x)=\beta(x)$ and $\operatorname{res} \xi(X)(\gamma)(x)=$ $\gamma(x)$. Since $\operatorname{res}_{\xi(X)}(\beta)=\operatorname{res} \xi(X)(\gamma)$, we have $(\alpha \circ \beta)=(\alpha \circ \gamma)$.

Now $f$ is a semigroup morphism since $\alpha \circ \beta \circ \alpha=\alpha \circ \beta$ for all covectors $\alpha$ and $\beta$ (see Proposition 3.4). To complete the proof observe that $f \circ g$ and $g \circ f$ are the identity morphisms of $\left.\mathcal{G}\right|_{\xi(X)}$ and $\mathcal{G}_{\geq \alpha}$, respectively.

\section{Structure of Oriented Interval Greedoids}

\section{$5.1 \mathcal{G}$ is a Graded Poset}

The next result generalizes [13, Lemma 4.1.12] from oriented matroids to oriented interval greedoids.

Lemma 5.1 Let $(E, \mathcal{F}, \mathcal{G})$ denote an oriented interval greedoid. Suppose $\alpha, \beta \in \mathcal{G}$ with $\operatorname{supp}(\alpha) \leq \operatorname{supp}(\beta)$ and $\alpha \not \leq \beta$. Then there exists $\delta \in \mathcal{G}$ such that $\delta \lessdot \beta$ and for all $x \notin S(\alpha, \beta)$, if $\beta(x) \neq 1$, then $\delta(x)=\beta(x)$.

Proof Let $A=\operatorname{supp}(\alpha)$ and $B=\operatorname{supp}(\beta)$. Suppose the result is not true. Of all $\alpha, \beta \in \mathcal{G}$ that violate the result choose a pair with $|S(\alpha, \beta)|$ minimal. If $S(\alpha, \beta)=\varnothing$, then $\alpha \leq \beta$ by Lemma 3.7, contradicting the assumption that $\alpha \not \leq \beta$. Therefore, $S(\alpha, \beta) \neq \varnothing$. Let $y \in S(\alpha, \beta)$. If $(\alpha \circ \beta)(y)=1$, then $(\beta \circ \alpha)(y)=1$ and so $\beta(y)=1$ because $A \leq B$ implies $(\beta \circ \alpha)=\beta$. This contradicts the fact that $y \in S(\alpha, \beta)$. Therefore, $(\alpha \circ \beta)(y) \neq 1$. (OG4) implies there exists $\gamma \in \mathcal{G}$ with $\gamma(y)=0$ and for all $x \notin S(\alpha, \beta)$, if $\beta(x)=(\beta \circ \alpha)(x) \neq 1$, then $\gamma(x)=(\alpha \circ \beta)(x)=(\beta \circ \alpha)(x)=\beta(x)$.

We argue that $S(\gamma, \beta) \subsetneq S(\alpha, \beta)$. Suppose $e \notin S(\alpha, \beta)$. Then either $\beta(e)=1$ or $\gamma(e)=\beta(e)$. In both cases $e \notin S(\gamma, \beta)$. Since $y \in S(\alpha, \beta)$ and $y \notin S(\gamma, \beta)$ (because $\gamma(y)=0)$, the inclusion is proper. 


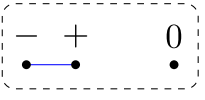

$\alpha$

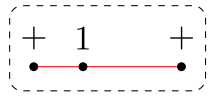

$\beta$

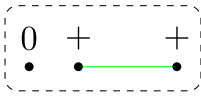

$\delta$

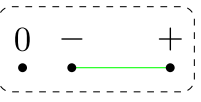

$\delta^{\prime}$

Fig. 7 The covectors $\delta$ and $\delta^{\prime}$ both satisfy the statement of Lemma 5.1 for the covectors $\alpha$ and $\beta$

Let $C=\operatorname{supp}(\gamma)$. We argue that $C<B$ by showing that $\beta(e)=0$ implies $\gamma(e)=0$ and that $B \neq C$. If $\beta(e)=0$, then $e \notin S(\alpha, \beta)$, so $\beta(e)=1$ (not possible) or $\gamma(e)=\beta(e)=0$. Since $\gamma(y)=0$ and $\beta(y) \in\{+,-\}$, we have $B \neq C$.

We argue that $S(\gamma, \beta) \neq \varnothing$. Suppose $S(\gamma, \beta)=\varnothing$. Then $\gamma<\beta$ by Lemma 3.7. Let $\delta \in \mathcal{G}$ denote a coatom in the interval $[\gamma, \beta]$ of the poset $\mathcal{G}$ and let $D=\operatorname{supp}(\delta)$. We will argue that $\delta$ satisfies the result, contradicting our assumption that no such $\delta$ exists. First note that $\delta \lessdot \beta$ by the choice of $\delta$. It remains to show that for all $x \notin S(\alpha, \beta)$, if $\beta(x) \neq 1$, then $\delta(x)=\beta(x)$. Let $x \notin S(\alpha, \beta)$. If $\beta(x) \neq 1$, then $\gamma(x)=\beta(x)$. Since $\gamma(x) \leq \delta(x) \leq \beta(x)$ and $\gamma(x)=\beta(x)$, it follows that $\delta(x)=\beta(x)$. And if $\beta(x)=1$, then $\gamma(x) \neq 0$, so $\delta(x) \geq \gamma(x)>0$.

We argued above that $C<B$ and $S(\gamma, \beta) \neq \varnothing$. Hence, $\gamma \not \leq \beta$ by Lemma 3.7. Since $\varnothing \neq S(\gamma, \beta) \subsetneq S(\alpha, \beta)$, the minimality of $|S(\alpha, \beta)|$ implies there exists $\delta \in \mathcal{G}$ such that $\delta \lessdot \beta$ and for all $x \notin S(\gamma, \beta)$, if $\beta(x) \neq 1$, then $\delta(x)=\beta(x)$. But since $S(\gamma, \beta) \subsetneq S(\alpha, \beta)$, if $x \notin S(\alpha, \beta)$, then $x \notin S(\gamma, \beta)$. Thus, for all $x \notin S(\alpha, \beta)$, if $\beta(x) \neq 1$, then $\delta(x)=\beta(x)$. This contradicts the assumption that no such $\delta$ exists for the pair $\alpha$ and $\beta$. We have arrived at a contradiction; so the result is true.

Example 5.2 Figure 7 illustrates Lemma 5.1 for the antimatroid corresponding to the convex geometry on three colinear points.

The partial order on the set of all covectors restricts to a partial order on $\mathcal{G}$. This next result shows that $\mathcal{G}$ is a graded poset and describes its rank function.

Proposition 5.3 Let $\mathcal{G}$ be an oriented interval greedoid over $(E, \mathcal{F})$. Then supp : $\mathcal{G} \rightarrow \Phi$ is a cover-preserving poset surjection of $\mathcal{G}$ onto $\Phi$ satisfying

$$
\operatorname{supp}(\alpha \circ \beta)=\operatorname{supp}(\alpha) \vee \operatorname{supp}(\beta) \text {. }
$$

In particular, $\mathcal{G}$ is graded of rank equal to the rank of $\Phi$. The rank of $\alpha \in \mathcal{G}$ is the rank of $\operatorname{supp}(\alpha) \in \Phi$.

Proof The identity follows immediately because if $A=\operatorname{supp}(\alpha)$ and $B=\operatorname{supp}(\beta)$, then $\operatorname{supp}(\alpha \circ \beta)=A \vee B$, by definition of the product. The fact that supp is a surjection of posets follows from its definition and axiom (OG1). It remains to show that supp is cover-preserving.

Suppose $\alpha \lessdot \beta$. Let $A=\operatorname{supp}(\alpha)$ and $B=\operatorname{supp}(\beta)$. Suppose there exists $C \in \Phi$ such that $A<C<B$. Let $\mathcal{G}^{\prime}=\left.\mathcal{G}\right|_{\xi(A)}$. Let $\alpha^{\prime}$ and $\beta^{\prime}$ be the elements of $\mathcal{G}^{\prime}$ corresponding to $\alpha, \beta$.

Since supp : $\mathcal{G}^{\prime} \rightarrow[A, \hat{1}]$ is surjective, there exists $\epsilon^{\prime} \in \mathcal{G}^{\prime}$ with $\operatorname{supp}\left(\epsilon^{\prime}\right)=C$. Let $\gamma^{\prime}=\alpha^{\prime} \circ \epsilon^{\prime}$. Then $\alpha^{\prime}<\gamma^{\prime}$ and $\operatorname{supp}\left(\gamma^{\prime}\right)=A \vee C=C$. If $\gamma^{\prime} \leq \beta^{\prime}$, then $\gamma^{\prime}=\beta^{\prime}$, 
contradicting that $C<B$. Hence, $\gamma^{\prime} \not \beta^{\prime}$. By Lemma 5.1 there exists $\delta^{\prime} \in \mathcal{G}^{\prime}$ such that $\delta^{\prime} \lessdot \beta^{\prime}$ and for all $x \notin S\left(\gamma^{\prime}, \beta^{\prime}\right)$, if $\beta^{\prime}(x) \neq 1$, then $\delta^{\prime}(x)=\beta^{\prime}(x)$. Let $D=$ $\operatorname{supp}\left(\delta^{\prime}\right)$. Since $\delta^{\prime} \in \mathcal{G}^{\prime}, A<D$. Thus $\alpha<\alpha \circ \delta<\alpha \circ \beta=\beta$ (see Proposition 3.4), contradicting that $\alpha \lessdot \beta$.

\subsection{Oriented Interval Greedoids of Rank 1}

Let $(E, \mathcal{F}, \mathcal{G})$ be an oriented interval greedoid and let $\Phi$ be its lattice of flats. The previous result shows that $\mathcal{G}$ is a graded lattice and that its rank is equal to that of $\Phi$. We define the rank of $(E, \mathcal{F}, \mathcal{G})$ to be the rank of $\mathcal{G}$ (equivalently, the rank of $\Phi$ ).

We first make a useful observation about arbitrary oriented interval greedoids.

Lemma 5.4 Suppose $(E, \mathcal{F}, \mathcal{G})$ is an oriented interval greedoid. Let $\hat{0}$ be the minimal element of $\Phi$. Then there is a unique element of $\mathcal{G}$ with support $\hat{0}$.

Proof By $(\mathrm{OG} 1)$, there exists $\varepsilon \in \mathcal{G}$ with $\operatorname{supp}(\varepsilon)=\hat{0}$. Then $\Gamma(\operatorname{supp}(\varepsilon))=\varnothing$, so $\varepsilon(e) \in\{0,1\}$ for all $e \in E$. Thus, $\varepsilon$ is determined by $\mathcal{F}$, and consequently is the unique element of $\mathcal{G}$ with support $\hat{0}$.

We will consistently denote the unique element of $\mathcal{G}$ with support $\hat{0}$ by $\varepsilon$.

The next result describes the oriented interval greedoids of rank 1.

Proposition 5.5 Suppose $(E, \mathcal{F}, \mathcal{G})$ is an oriented interval greedoid of rank 1 . Then $\mathcal{G}$ contains exactly three elements, and its Hasse diagram is

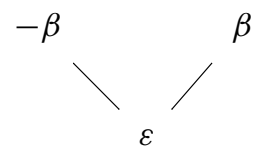

Proof Since $\mathcal{G}$ has rank 1, $\Phi$ contains exactly two elements, its minimal and maximal elements $\hat{0}$ and $\hat{1}=[\varnothing]$, respectively.

By the previous lemma, $\varepsilon$ is the unique element of $\mathcal{G}$ with support $\hat{0}$. We now show that there exist exactly two elements in $\mathcal{G}$ of support $\hat{1}$. By (OG1), there exists $\beta \in \mathcal{G}$ such that $\operatorname{supp}(\beta)=\hat{1}$. Then $-\beta \in \mathcal{G}$ by $(\mathrm{OG} 2)$. Since $\Gamma(\varnothing) \neq \varnothing, \beta \neq-\beta$. So $\mathcal{G}$ contains at least two elements of support $\hat{1}$.

Let $\alpha \in \mathcal{G}, \alpha \neq \beta$ and $\operatorname{supp}(\alpha)=\hat{1}$. Let $y \in \Gamma(\varnothing), y \notin S(\alpha, \beta)$. Then let $\delta$ be the vector guaranteed by Lemma 5.1. Then $\delta(y)=\beta(y)$. But $\delta=\varepsilon$, so this is impossible. It follows that $S(\alpha, \beta)=\Gamma(\varnothing)$; in other words, $\alpha=-\beta$.

\subsection{Oriented Interval Greedoids of Rank 2}

Proposition 5.6 Suppose $(E, \mathcal{F}, \mathcal{G})$ is an oriented interval greedoid of rank 2 . Then $\mathcal{G}$ is isomorphic to the semigroup of covectors of an oriented matroid of rank 2 , or 
the Hasse diagrams of $\mathcal{G}$ and $\Phi$ are, respectively, the following two posets.
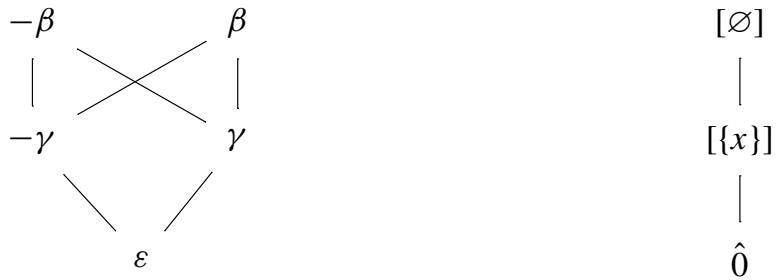

Proof There are two cases to consider.

Case 5.6.1 Suppose $\Phi$ contains at least two coatoms. By Proposition 4.12, the restriction $\left.\mathcal{G}\right|_{\Gamma(\varnothing)}$ is an oriented matroid. The map $C \mapsto[Y]$ for any $Y \in C$ embeds $\left.\Phi\right|_{\Gamma(\varnothing)}$ into the interval [[X], $\left.\hat{1}\right]$ of $\Phi$, where $X$ is the maximal among the feasible sets contained in $\Gamma(\varnothing)$ (see Sect. 4.2.1). Since every coatom of $\Phi$ is of the form $[\{x\}]$ for some $x \in \Gamma(\varnothing)$, there is a bijection between the coatoms of $\Phi$ and those of $\left.\Phi\right|_{\Gamma(\varnothing)}$. Therefore, $[X]=\hat{0}$, so $\left.\Phi\right|_{\Gamma(\varnothing)} \cong \Phi$. So $\left.\mathcal{G}\right|_{\Gamma(\varnothing)}$ is a rank 2 oriented matroid. We argue that the map $\left.\gamma \mapsto \gamma\right|_{\Gamma(\varnothing)}$ is an isomorphism $\left.\mathcal{G} \cong \mathcal{G}\right|_{\Gamma(\varnothing)}$. By Proposition 4.12 we need only show that this is an injection.

Let $\gamma, \gamma^{\prime} \in \mathcal{G}$ and suppose $\left.\gamma\right|_{\Gamma(\varnothing)}=\left.\gamma^{\prime}\right|_{\Gamma(\varnothing)}$. Then $\left.\operatorname{supp}(\gamma)\right|_{\Gamma(\varnothing)}=\left.\operatorname{supp}\left(\gamma^{\prime}\right)\right|_{\Gamma(\varnothing)}$. Since $\left.\Phi\right|_{\Gamma(\varnothing)} \cong \Phi$, it follows that $\operatorname{supp}(\gamma)=\operatorname{supp}\left(\gamma^{\prime}\right)$. This implies that $\gamma(x)$ is 0 or 1 if and only if $\gamma^{\prime}(x)$ is 0 or 1 , respectively. Let $C=\operatorname{supp}(\gamma)=\operatorname{supp}\left(\gamma^{\prime}\right)$.

If $C=\hat{0}$, then $\gamma=\gamma^{\prime}$ since there is a unique element of $\mathcal{G}$ with support $\hat{0}$. If $C=\hat{1}=[\varnothing]$, then $\left.\gamma\right|_{\Gamma(\varnothing)}=\left.\gamma^{\prime}\right|_{\Gamma(\varnothing)}$ implies that $\gamma=\gamma^{\prime}$ since they agree on $\Gamma(\varnothing)$.

Let $C \rightarrow \hat{0}$. Suppose $\gamma \neq \gamma^{\prime}$. Arguing as in the end of Proposition 5.5, we conclude $\Gamma(C)=S\left(\gamma, \gamma^{\prime}\right)$. Since $\gamma$ and $\gamma^{\prime}$ agree on $\Gamma(\varnothing)$ and disagree on $\Gamma(C)$, it follows $\left.\gamma\right|_{\Gamma(\varnothing)}=\left.\gamma^{\prime}\right|_{\Gamma(\varnothing)}$ takes values in $\{0,1\}$. Thus, $\left.C\right|_{\Gamma(\varnothing)}=\hat{0}$, which implies $C=\hat{0}$, contradicting that $C>\hat{0}$. Thus, $\gamma=\gamma^{\prime}$.

Case 5.6.2 Suppose $\Phi$ contains exactly one coatom. Then $[X]$ is this coatom, so $X=\{x\}$ for some $x \in E$. By Lemma $5.4, \varepsilon$ is the unique element of $\mathcal{G}$ with support $\hat{0}$.

By (OG1), there exists $\gamma \in \mathcal{G}$ such that $\operatorname{supp}(\gamma)=[X]$. By arguing as in Proposition 5.5, we conclude that $\gamma \neq-\gamma$ and that if $v \in \mathcal{G}$ with $\operatorname{supp}(v)=[X]$, then $v=\gamma$ or $v=-\gamma$. Hence, there are exactly two elements in $\mathcal{G}$ of support $[X]$.

By (OG1), there exists $\beta \in \mathcal{G}$ such that $\operatorname{supp}(\beta)=[\varnothing]$. By $(\mathrm{OG} 2)-\beta \in \mathcal{G}$. As above, we have $\beta \neq-\beta . \Gamma(\varnothing) \cap \Gamma(X)=\varnothing$, since if $y \in \Gamma(\varnothing) \cap \Gamma(X)$, then $y \notin$ $\xi(X)$, so $[y] \neq[x]$, contradicting our assumption that $\Phi$ has only one coatom. Thus $\gamma,-\gamma<\beta,-\beta$.

Let $v \in \mathcal{G}$ such that $\operatorname{supp}(v)=\hat{1}$. By arguing as before (using Lemma 5.1), it follows that $\nu=\beta$ or $\nu=-\beta$.

\subsection{Intervals of Length Two}

Let $\hat{\mathcal{G}}$ denote the poset obtained from $\mathcal{G}$ by adjoining a maximal element $\hat{1}$. We prove that all intervals of length two in $\hat{\mathcal{G}}$ contain exactly four elements. 
Proposition 5.7 Suppose $\mathcal{G}$ is an oriented interval greedoid. Then all intervals in $\hat{\mathcal{G}}$ of length two contain exactly four elements.

Proof Let $\alpha, \beta, \gamma \in \hat{\mathcal{G}}$ such that $\alpha \lessdot \gamma \lessdot \beta$. The case where $\beta=\hat{1}$ was proved in Proposition 5.5, so suppose $\beta \in \mathcal{G}$. Let $A=\operatorname{supp}(\alpha), B=\operatorname{supp}(\beta)$ and $C=\operatorname{supp}(\gamma)$. By Proposition 5.3, $A \lessdot C \lessdot B$ in $\Phi$.

Let $\operatorname{supp}(\alpha)=[X]$ for some $X \in \mathcal{F}$. By Proposition 4.16 and Proposition 3.4, $\left.\mathcal{G}\right|_{\xi(X)} \cong \mathcal{G}_{\geq \alpha}$ (as posets), so $\{\delta \in \mathcal{G}: \alpha \lessdot \delta \lessdot \beta\} \cong\left\{\left.\delta \in \mathcal{G}\right|_{\xi(X)}:\left.\left.\alpha\right|_{\xi(X)} \lessdot \delta \lessdot \beta\right|_{\xi(X)}\right\}$. Thus, by passing to $\left.\mathcal{G}\right|_{\xi(X)}$ we can suppose that $A=\hat{0}$.

Let $\operatorname{supp}(\beta)=[Y]$ for some $Y \in \mathcal{F}$. By Proposition 4.4, $\mathcal{G} / Y \cong \mathcal{G}_{\leq[Y]}=\{v \in \mathcal{G}$ : $\operatorname{supp}(v) \leq[Y]\}$. Since $\Phi / Y \cong[\hat{0},[Y]] \subseteq \Phi$ (Proposition 4.1), by passing to $\mathcal{G} / Y$, we can suppose that $B=\hat{1}$, and therefore that $\Phi$ is a lattice of rank 2 .

Proposition 5.6 classified the oriented interval greedoids of rank 2 as being either an oriented matroid of rank 2 or having the Hasse diagram shown in the statement of Proposition 5.6. For the latter situation a quick inspection of the given poset establishes the result. And for the former situation, it is well-known that this result holds for oriented matroids [13, Theorem 4.1.14].

\subsection{The Underlying Oriented Matroid}

Let $(E, \mathcal{F}, \mathcal{G})$ be an oriented interval greedoid. The top element in the poset of flats $\Phi$ is $[\varnothing]$, and by Proposition 3.3 it follows that $\Gamma(\varnothing) \subseteq \Gamma(A) \cup \xi(A)$ for any flat $A \in \Phi$. This implies that $\alpha(x) \in\{0,+,-\}$ for any $\alpha \in \mathcal{G}$ and any $x \in \Gamma(\varnothing)$. Moreover, $\Gamma(\varnothing)$ is the largest subset of $E$ with this property: if $\alpha$ is maximal in $\mathcal{G}$, then $\operatorname{supp}(\alpha)=[\varnothing]$ and $\alpha(x)=1$ if and only if $x \notin \Gamma(\varnothing)$. This observation implies that the restriction to $\Gamma(\varnothing)$ produces an oriented interval greedoid whose covectors take values in $\{0,+,-\}$. Thus, $\left.\mathcal{G}\right|_{\Gamma(\varnothing)}$ is an oriented matroid. Alternatively, one can note that the restriction $\left(\Gamma(\varnothing),\left.\mathcal{F}\right|_{\Gamma(\varnothing)}\right)$ is a matroid and appeal to Theorem 3.12.

Definition 5.8 Let $\mathcal{G}$ denote an oriented interval greedoid over $(E, \mathcal{F})$. The underlying oriented matroid of $\mathcal{G}$ is $\overline{\mathcal{G}}=\left.\mathcal{G}\right|_{\Gamma(\varnothing)}$.

The lattice of flats $\bar{\Phi}$ of $\overline{\mathcal{G}}$ is a geometric lattice because $\overline{\mathcal{G}}$ is an oriented matroid. Moreover, it is isomorphic to the sublattice of $\Phi$ generated by all the coatoms.

\subsection{The Tope Graph}

A tope of an oriented interval greedoid $(E, \mathcal{F}, \mathcal{G})$ is a covector that is maximal in $\mathcal{G}$ with respect to the partial order on covectors. Alternatively, topes are covectors whose support is $\hat{1}=[\varnothing]$. A subtope of $\mathcal{G}$ is a covector in $\mathcal{G}$ that is covered by some tope. From Proposition 5.7 it follows that every subtope is covered by exactly two different topes. Two topes are said to be adjacent if there exists a subtope that is covered by both topes.

The tope graph $\mathrm{T}(\mathcal{G})$, or just $\mathrm{T}$, of $\mathcal{G}$ is the graph with one vertex for each tope of $\mathcal{G}$ and an edge between two vertices if the corresponding topes are adjacent. 
Lemma 5.9 Suppose $\mathcal{G}$ is an oriented interval greedoid. Then the tope graph of $\mathcal{G}$ is isomorphic to the tope graph of the underlying oriented matroid $\overline{\mathcal{G}}$ of $\mathcal{G}$.

Proof First we will show that topes of $\mathcal{G}$ are in one-to-one correspondence with the topes of $\overline{\mathcal{G}}$. Suppose $\alpha$ is a tope in $\mathcal{G}$. Then $\operatorname{supp}(\alpha)=[\varnothing]=\{\varnothing\}$, and so $\left.\operatorname{supp}\right|_{\Gamma(\varnothing)}\left(\operatorname{res}_{\Gamma(\varnothing)}(\alpha)\right)=\{\varnothing\}=\left.\hat{1} \in \Phi\right|_{\Gamma(\varnothing)}$. Thus, $\operatorname{res}_{\Gamma(\varnothing)}(\alpha)$ is a tope of $\overline{\mathcal{G}}$.

Conversely, suppose $\operatorname{res}_{\Gamma(\varnothing)}(\alpha)$ is a tope of $\overline{\mathcal{G}}$. Then $\left.\operatorname{supp}\right|_{\Gamma(\varnothing)}\left(\operatorname{res}_{\Gamma(\varnothing)}(\alpha)\right)=$ $\left.[\varnothing]\right|_{\Gamma(\varnothing)}=\{\varnothing\}$. Let $A=\operatorname{supp}(\alpha)$. Then $\left.A\right|_{\Gamma(\varnothing)}=\{\varnothing\}$, so $\varnothing$ is maximal among the feasible sets contained in $\Gamma(\varnothing) \cap \xi(A)$. This implies that $\Gamma(\varnothing) \cap \xi(A)=\varnothing$ (if $x \in \Gamma(\varnothing) \cap \xi(A)$, then $\{x\} \in \mathcal{F}$ because $x \in \Gamma(\varnothing)$, contradicting that maximality of $\varnothing$ ). If $A \neq[\varnothing]$, then $A \leq[\{y\}]$ for some $y \in \Gamma(\varnothing)$. Hence, $y \in \Gamma(\varnothing) \cap \xi(A)$, contradicting that $\Gamma(\varnothing) \cap \xi(A) \neq \varnothing$. Thus, $A=[\varnothing]$. So $\alpha$ is a tope of $\mathcal{G}$.

Let $\alpha$ and $\beta$ be topes in $\mathcal{G}$ and suppose $\operatorname{res}_{\Gamma(\varnothing)}(\alpha)=\operatorname{res}_{\Gamma(\varnothing)}(\beta)$. We show that $\alpha=\beta$ by showing that they agree on $\Gamma(\operatorname{supp}(\alpha))=\Gamma(\operatorname{supp}(\beta))=\Gamma(\varnothing)$. Since $\operatorname{supp}(\alpha)=\operatorname{supp}(\beta)=[\varnothing]$, we have $\Gamma(\varnothing)=\left.\Gamma\right|_{\Gamma(\varnothing)}(\varnothing)=\left.\Gamma\right|_{\Gamma(\varnothing)}\left(\left.[\varnothing]\right|_{\Gamma(\varnothing)}\right)$. Hence, $\operatorname{res}_{\Gamma(\varnothing)}(\alpha)(w)=\alpha(w)$ and $\operatorname{res}_{\Gamma(\varnothing)}(\beta)(w)=\beta(w)$ for all $w \in \Gamma(\varnothing)$. It follows that $\alpha(w)=\beta(w)$ for all $w \in \Gamma(\varnothing)$. This establishes the one-to-one correspondence.

Suppose $\alpha, \beta \in \mathcal{G}$ are two adjacent topes and let $\gamma \in \mathcal{G}$ with $\gamma \lessdot \alpha$, $\beta$. Then $\operatorname{supp}(\gamma) \lessdot \operatorname{supp}(\alpha)=\operatorname{supp}(\beta)=[\varnothing]$. Since $\operatorname{res}_{\Gamma(\varnothing)}$ is a semigroup morphism, it follows that $\operatorname{res}_{\Gamma(\varnothing)}(\gamma) \leq \operatorname{res}_{\Gamma(\varnothing)}(\alpha)$. We cannot have equality since this would imply that both are topes of $\left.\mathcal{G}\right|_{\Gamma(\varnothing)}$, contradicting that $\gamma$ is not a tope. We have $\operatorname{supp}(\gamma)=[\{y\}]$ for some $y \in \Gamma(\varnothing)$ since all coatoms of $\Phi$ are of this form. Hence, $\operatorname{res}_{\Gamma(\varnothing)}(\gamma)=\left.\left.[\{y\}]\right|_{\Gamma(\varnothing)} \lessdot[\varnothing]\right|_{\Gamma(\varnothing)}$. Since supp $\left.\right|_{\Gamma(\varnothing)}$ is cover-preserving, it follows

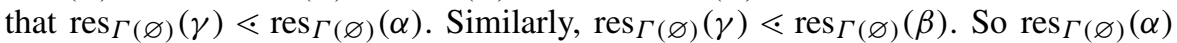
and $\operatorname{res}_{\Gamma(\varnothing)}(\beta)$ are adjacent topes.

Let $\operatorname{res}_{\Gamma(\varnothing)}(\alpha),\left.\operatorname{res}_{\Gamma(\varnothing)}(\beta) \in \mathcal{G}\right|_{\Gamma(\varnothing)}$ be adjacent topes and let $\operatorname{res}_{\Gamma(\varnothing)}(\gamma) \in$ $\left.\mathcal{G}\right|_{\Gamma(\varnothing)}$ with $\operatorname{res}_{\Gamma(\varnothing)}(\gamma) \in \mathcal{G}$ with $\operatorname{res}_{\Gamma(\varnothing)}(\gamma) \lessdot \operatorname{res}_{\Gamma(\varnothing)}(\alpha), \operatorname{res}_{\Gamma(\varnothing)}(\beta)$. Since $\operatorname{res}_{\Gamma(\varnothing)}(\gamma \circ \alpha)=\operatorname{res}_{\Gamma(\varnothing)}(\gamma) \circ \operatorname{res}_{\Gamma(\varnothing)}(\alpha)=\operatorname{res}_{\Gamma(\varnothing)}(\alpha)$ and since $\gamma \circ \alpha$ and $\alpha$ are both topes, we have $\alpha=\gamma \circ \alpha$. So $\gamma<\alpha$. To show that $\gamma \lessdot \alpha$, it suffices to show that $\operatorname{supp}(\gamma) \lessdot[\varnothing]$. Let $C=\operatorname{supp}(\gamma)$. If $C$ is not covered by $[\varnothing]$, then $C \leq[\{x, y\}]$ for some $x, y \in \Gamma(\varnothing), x \neq y$. Thus, $\{x, y\} \subseteq \Gamma(\varnothing) \cap \xi(C)$. Let $Y \supseteq\{x, y\}$ be maximal among the feasible sets contained in $\xi(C) \cap \Gamma(\varnothing)$. By definition, supp $\left.\right|_{\Gamma(\varnothing)}\left(\operatorname{res}_{\Gamma(\varnothing)}(\gamma)\right)=\left.C\right|_{\Gamma(\varnothing)}$ is the flat containing $Y$. Since $|Y|>2$, it follows that supp $\left.\right|_{\Gamma(\varnothing)}\left(\operatorname{res}_{\Gamma(\varnothing)}(\gamma)\right)$ is not a coatom of $\left.\Phi\right|_{\Gamma(\varnothing)}$, contradicting that it is. Hence, $\gamma \lessdot \alpha$. Similarly, $\gamma \lessdot \beta$. Therefore, $\alpha$ and $\beta$ are adjacent topes.

\section{CW-Spheres from Oriented Interval Greedoids}

\subsection{CW-Spheres}

The Sphericity Theorem is an important result for oriented matroids which asserts that there is a certain regular $\mathrm{CW}$-sphere associated to any oriented matroid, whose cells correspond to the covectors of the oriented matroid. It is originally due to Folkman and Lawrence [21]; see also [13, Theorem 4.3.3]. In this section and the next, we will prove the corresponding result for oriented interval greedoids. 
We recall some topological definitions, following [13, Sect. 4.7].

A ball is a topological space homeomorphic to the usual closed $d$-dimensional ball, for some nonnegative integer $d$.

A regular cell complex $\Delta$ is a finite set of balls in a Hausdorff topological space $|\Delta|=\bigcup_{\sigma \in \Delta} \sigma$ with the properties that:

- The interiors of the balls $\sigma \in \Delta$ partition $|\Delta|$.

- For each $\sigma \in \Delta$, the boundary of $\sigma$ is the union of some elements $\tau \in \Delta$.

This definition of a regular cell complex is (non-trivially) equivalent to the usual definition of a regular CW-complex. (See [13, Sect. 4.7].)

A cell complex $\Delta$ is called a regular $C W$-sphere if its geometric realization $|\Delta|$ is homeomorphic to a sphere.

The face poset of a cell complex is the poset structure on the cells of $\Delta$, ordered by containment. The augmented face poset of a cell complex is the face poset with a maximal element $\hat{1}$ adjoined.

We can now state our main theorem for this section more precisely.

Theorem 6.1 For $(E, \mathcal{F}, \mathcal{G})$ an oriented interval greedoid, $\hat{\mathcal{G}}$ is isomorphic to the augmented face poset of a regular $C W$-sphere.

In the oriented matroid setting, a trivial case of the Sphericity Theorem is when the oriented matroid arises from a hyperplane arrangement (with, say, all the hyperplanes containing the origin): taking a small sphere about the origin, and thinking of it as sliced into pieces by the hyperplanes, one immediately obtains a regular $\mathrm{CW}$-sphere as in the statement of the theorem. Similarly, complexified hyperplane arrangements (see Sect. 3.4.3) yield examples where it is clear that the above theorem holds, and which can serve as useful illustrations.

Example 6.2 A more interesting example is the following. We construct a greedoid on $E=\{a, b, c, e\}$. Let $F=\{a, b, c, a b, a c, b c\}$. Let the feasible sets be as follows:

$$
\mathcal{F}=\{\varnothing\} \cup F \cup\{X \cup\{e\} \mid X \in F\} .
$$

Consider a two-dimensional sphere, draw three great circles on it, each passing through the north and south poles, and mark the points where each great circle crosses the equator. The result is a regular CW-sphere with 6 two-cells, 12 one-cells, and 8 zero-cells, and it is clear how the cells correspond to a collection of covectors (which do indeed form an oriented interval greedoid structure for $(E, \mathcal{F})$ ).

This example can be generalized to start with $F$ being the non-empty feasible sets of an arbitrary matroid; then any oriented structure for that matroid gives rise to an oriented interval greedoid structure for the corresponding greedoid.

The order complex of a bounded poset $P$ is the simplicial complex consisting of chains in $P \backslash\{\hat{0}, \hat{1}\}$. Taking a barycentric subdivision of the $\mathrm{CW}$-sphere in the previous theorem, we obtain the following.

Corollary 6.3 The order complex of $\hat{\mathcal{G}}$ is a simplicial sphere. 
Proof of Theorem 6.1 As in the proof of the Sphericity Theorem in [13], the main technical tool required in the proof is the notion of recursive coatom ordering.

Let $P$ be a graded, bounded poset. A linear ordering of its coatoms, $q_{1}, \ldots, q_{r}$, is called a recursive coatom ordering if it satisfies:

(i) $\left[\hat{0}, q_{i}\right]$ admits a recursive coatom ordering in which the coatoms of $\left[\hat{0}, q_{i}\right]$ which lie below some $q_{j}$ with $j<i$, come first;

(ii) any element lying below $q_{i}$ and also below some $q_{j}$ for $j<i$, necessarily lies below a coatom of $\left[\hat{0}, q_{i}\right]$ which lies below some $q_{k}$ with $k<i$.

The unique linear order on the (single) coatom of a bounded poset of rank 1 is considered to be a recursive coatom ordering, so any linear order on the coatoms of a bounded poset of rank 2 is a recursive coatom ordering. Recursive coatom orderings are dual to recursive atom orderings, which go back to [16]. The concept has been extended to non-graded posets [17], but we shall not need that here.

The fundamental technical result is the following lemma, whose proof we defer to Sect. 6.2.

\section{Lemma $6.4 \hat{\mathcal{G}}$ admits a recursive coatom ordering.}

A poset is called thin if all intervals of length 2 have cardinality four. By Proposition 5.7, we know that $\hat{\mathcal{G}}$ is thin. The following theorem completes our proof.

Theorem 6.5 ([9], [13, Theorem 4.7.24]) $P$ is isomorphic to the face poset of a shellable regular cell decomposition of the sphere iff $P$ is thin and admits a recursive coatom ordering.

(We shall not discuss the significance of the "shellable" in the above theorem; the interested reader is directed to [13].)

We now turn to the proof of the corollary.

Proof of Corollary 6.3 The order complex of the augmented face poset of a regular cell complex $\Delta$ is homeomorphic to $|\Delta|$ [13, Proposition 4.7.8]. (In fact, the order complex should be thought of as the barycentric subdivision of the regular cell complex.) The corollary follows.

\subsection{A Recursive Coatom Ordering for $\hat{\mathcal{G}}$}

This section is devoted to the proof of Lemma 6.4, which asserts that $\hat{\mathcal{G}}$ has a recursive coatom ordering.

If one chooses a particular tope $\alpha$ of $\mathcal{G}$ then there is a natural poset structure on the topes with respect to which $\alpha$ is the minimum element and $-\alpha$ is the maximum element, and the Hasse diagram is (a suitable orientation of) the tope graph. This poset is called $\mathcal{T}(\mathcal{G}, \alpha)$. (Since the topes of $\mathcal{G}$ are identified with the topes of $\overline{\mathcal{G}}$, this follows from the analogous statements for oriented matroids; see [13, Sect. 4.2].)

Let $\alpha$ be a tope of $\mathcal{G}$. Consider a maximal chain $\bar{\beta}$ in $\mathcal{T}(\mathcal{G}, \alpha)$, say $\alpha=\beta_{0}<\cdots<$ $\beta_{r}=-\alpha$. Choose $\gamma_{i}$ to be a common facet of $\beta_{i-1}$ and $\beta_{i}$. Let $G_{i}=\operatorname{supp}\left(\gamma_{i}\right)$. The 
$G_{i}$ are distinct and include all the coatoms of $\Phi$. Thus, $\bar{\beta}$ induces a linear order on the coatoms of $\Phi$. However (unlike the situation for oriented matroids) this does not immediately yield a linear order on the coatoms of $[\varepsilon, \alpha]$, because there may be more than one coatom with the same support.

For $1 \leq i \leq r$, let $\mathcal{G}_{i}$ be the oriented matroid obtained by contracting $\mathcal{G}$ to $G_{i}$. Consider the tope poset $\mathcal{T}\left(\mathcal{G}_{i}, \gamma_{i}\right)$.

Let $\Delta$ be the set of facets of $\alpha$. Let $\Delta_{i}$ be the set of facets of $\alpha$ whose support is $G_{i}$. (This set could be empty.)

A linear extension of $\mathcal{T}\left(\mathcal{G}_{i}, \gamma_{i}\right)$ will be called adapted to $\alpha$ if it contains in order:

(1) first, the topes of $\mathcal{G}_{i}$ that lie on the same side as $\gamma_{i}$ of some $G_{j}$ for $j<i$,

(2) then, the topes that are facets of $\alpha$,

(3) finally, the remaining topes of $\mathcal{G}_{i}$.

We will need the following lemma:

Lemma 6.6 $\mathcal{T}\left(\mathcal{G}_{i}, \gamma_{i}\right)$ admits a linear extension adapted to $\alpha$.

Proof It is certainly possible to define a linear extension of $\mathcal{T}\left(\mathcal{G}_{i}, \gamma_{i}\right)$ which begins with the elements (1) above, since they form a lower order ideal in $\mathcal{T}\left(\mathcal{G}_{i}, \gamma_{i}\right)$. In order to be able to construct a linear extension such that the next elements are those from (2) above, we need to show that any tope below a tope from (2) not in (2), is contained in (1). If $\delta$ is a tope of $\Delta_{i}$ which is a facet of $\alpha$, and $\epsilon$ is a tope lying below $\delta$ which is not a facet of $\alpha$, it must be separated from $\alpha$ by some $G_{j}$ with $j<i$, which shows that $\epsilon$ is in (1). Thus the linear extension, whose beginning was already described, can be continued with the set of facets of $\alpha$, followed by the remaining topes of $\mathcal{G}_{i}$.

A linear order on $\Delta$ will be said to be compatible with $\bar{\beta}$ if

(1) the elements of $\Delta$ are arranged first of all in increasing order by support (so $\Delta_{1}$ comes first, then $\Delta_{2}$, etc.),

(2) the elements of $\Delta_{i}$ are arranged according to a linear order on $\mathcal{T}\left(\mathcal{G}_{i}, \gamma_{i}\right)$ which is adapted to $\alpha$.

Now we will prove the following:

\section{Proposition 6.7}

(1) For a tope $\alpha$ in $\mathcal{G}$, and a maximal chain $\bar{\beta}$ in $\mathcal{T}(\mathcal{G}, \alpha)$, any order on the coatoms of $[\varepsilon, \alpha]$ compatible with $\bar{\beta}$ is a recursive coatom order.

(2) For a tope $\alpha$ in $\mathcal{G}$, any linear extension of $\mathcal{T}(\mathcal{G}, \alpha)$ is a recursive coatom ordering for $\hat{\mathcal{G}}$.

Proof The proof will be by induction on the rank of $\mathcal{G}$. The base case, when the rank of $\mathcal{G}$ is 1 , is trivial. We will assume that (1) and (2) hold for oriented interval greedoids of rank less than $n$; we will prove (1) for oriented interval greedoids of rank $n$, and then make use of (1) to prove (2) for oriented interval greedoids of rank $n$.

Proof of (1) Pick a coatom order for $[\varepsilon, \alpha]$ which is compatible with $\bar{\beta}$. As part of this, we are given $\gamma_{i}$ a common facet of $\beta_{i-1}$ and $\beta_{i}$. Let $G_{i}$ be the support of $\gamma_{i}$. 
Let $\Delta_{i}$ be the coatoms of $\alpha$ with support $G_{i}$. As part of our coatom order for $[\varepsilon, \alpha]$, we are given a linear order on $\Delta_{i}$ which is the restriction of a linear extension of $\mathcal{T}\left(\mathcal{G}_{i}, \gamma_{i}\right)$ adapted to $\alpha$. Fix such a linear extension.

Let $\delta \in \Delta_{i}$ be a coatom of $[\varepsilon, \alpha]$. We must define a coatom order for $[\varepsilon, \delta]$. Using our chosen linear extension of $\mathcal{T}\left(\mathcal{G}_{i}, \gamma_{i}\right)$, we can apply (2) to $\hat{\mathcal{G}}_{i}$, obtaining a recursive coatom order for $[\varepsilon, \delta]$. We must show that this order satisfies the necessary conditions.

Now, $\delta$ is a coatom of two different posets, $[\varepsilon, \alpha]$ and $\hat{\mathcal{G}}_{i}$. Let $X$ be the set of coatoms of $[\varepsilon, \alpha]$ which precede $\delta$ with respect to the coatom order on $[\varepsilon, \alpha]$, and let $Y$ be the set of coatoms of $\hat{\mathcal{G}}_{i}$ which precede $\delta$ with respect to the fixed linear extension of $\mathcal{T}\left(\mathcal{G}_{i}, \gamma_{i}\right)$. Let $\check{X}$ be the coatoms of $[\varepsilon, \delta]$ lying below an element of $X$, and let $\check{Y}$ be the coatoms of $[\varepsilon, \delta]$ lying below an element of $Y$. We will now show that $\check{X}$ and $\check{Y}$ coincide.

Let $\epsilon$ be a coatom of $[\varepsilon, \delta]$. By Proposition $4.16, \mathcal{G}_{\geq \epsilon}$ is itself an oriented greedoid, so we may assume that $\epsilon=\varepsilon$, or, in other words, that $\mathcal{G}$ is rank 2. By Proposition 5.6, we know that $\mathcal{G}$ is either isomorphic to a rank 2 oriented matroid, or else it is of the special form described in that proposition. In either case, it is straightforward to check that $\epsilon \in \check{X}$ iff $\epsilon \in \check{Y}$.

Since we know property (i) of recursive coatom orders holds for our fixed linear extension of $\mathcal{T}\left(\mathcal{G}_{i}, \gamma_{i}\right)$, property (i) also follows for our coatom ordering on $[\varepsilon, \alpha]$.

Next, we check property (ii). Let $\epsilon \in[\varepsilon, \delta]$, which lies under some element $\zeta \in X$. We must show that it also lies below some element of $\check{X}$.

Again, by restricting, we may assume that $\epsilon=\varepsilon$. The fact that $\epsilon$ lies under an element of $X$ implies, in particular, that $X$ is non-empty, and thus that $\delta$ is not the first coatom in our coatom order on $[\varepsilon, \alpha]$. We will now show that $Y$ is non-empty. If $\delta$ is not the first coatom with support $G_{i}$ in our recursive coatom order on $[\varepsilon, \alpha]$ then this is clear. So suppose that $\delta$ is the first coatom with support $G_{i}$ in our recursive coatom order. Since $\delta$ is not the first coatom overall, it must be that $i>1$. Therefore $\gamma_{i}$ is not a facet of $\alpha$, so $\gamma_{i} \in Y$.

Now, since we have assumed that $\epsilon=\varepsilon$, the fact that $Y$ is non-empty means that there are elements of $Y$ lying over $\epsilon$. Therefore, by property (ii) for the fixed linear extension of $\mathcal{T}\left(\mathcal{G}_{i}, \gamma_{i}\right)$, we know that there are elements of $\check{Y}$ lying over $\epsilon$. Since $\check{Y}=\check{X}$, we are done.

Proof of (2), assuming (1) Pick a linear extension of $\mathcal{T}(\mathcal{G}, \alpha)$. For each coatom $\delta$ of $\hat{\mathcal{G}}$, pick a maximal chain $\bar{\beta}$ in $\mathcal{T}(\mathcal{G}, \delta)$ which includes $\alpha$. Then we claim that any linear order on the coatoms of $[\varepsilon, \delta]$, compatible with $\bar{\beta}$, satisfies the necessary conditions. First of all, it is a recursive coatom order by (1).

Second, define $Q_{\delta}$ to be the set of coatoms of $[\varepsilon, \delta]$ which also lie under some $\xi$ preceding $\delta$ in the linear extension of $\mathcal{T}(\mathcal{G}, \alpha)$. The coatoms of $Q_{\delta}$ precede the other coatoms of $\delta$ in any order compatible with $\bar{\beta}$. (In fact, for this, it suffices to know that an order compatible with $\bar{\beta}$ agrees with the order induced by $\bar{\beta}$ on the coatoms of $[\hat{0}, \operatorname{supp}(\delta)]$.) This proves (i).

Thirdly, we check that

$$
\bigcup_{\zeta \in Q_{\delta}}[\varepsilon, \zeta]=[\varepsilon, \delta] \cap \bigcup_{\xi \text { preceding } \delta}[\varepsilon, \xi] .
$$


The containment of the left-hand side in the right-hand side is obvious. For the opposite inclusion, let $\epsilon \in[\varepsilon, \delta] \cap[\varepsilon, \xi]$ for some $\xi$ preceding $\delta$. The topes of $\mathcal{G}$ that contain $\epsilon$ are exactly the topes of $\overline{\mathcal{G}}$ that contain $\left.\epsilon\right|_{\Gamma(\varnothing)}$. By [13, Lemma 4.2.12], this is an interval $I$ in $\mathcal{T}(\overline{\mathcal{G}}, \alpha)$. Since $\epsilon$ is contained in some $\xi$ preceding $\delta$, we know that $\delta$ is not the minimum element of the interval. Let $\rho$ be covered by $\delta$ in $I$. Since $\rho$ lies below $\delta$ in $\mathcal{T}(\mathcal{G}, \alpha)$, it precedes $\delta$ in the linear extension of $\mathcal{T}(\mathcal{G}, \alpha)$. Since $\rho$ is in $I$, $\epsilon \in[\hat{0}, \rho]$. Finally, since $\rho$ and $\delta$ are adjacent topes, they have a common subtope $\sigma$ in $\overline{\mathcal{G}}$. Since, in $\hat{\mathcal{G}}, \rho$ and $\delta$ lie over $\left.\epsilon\right|_{\Gamma(\varnothing)}, \sigma$ lies over $\left.\epsilon\right|_{\Gamma(\varnothing)}$. Thus $\operatorname{supp}(\sigma)$ lies over $\operatorname{supp}(\epsilon)$.

Let $\phi$ be covector of $\mathcal{G}$, such that $\left.\phi\right|_{\Gamma(\varnothing)}=\sigma$. Now consider $\epsilon \circ \phi$. This lies over $\epsilon$, and its $\operatorname{support}$ is $\operatorname{supp}(\epsilon) \vee \operatorname{supp}(\phi)=\operatorname{supp}(\phi)$. Since, in $\hat{\mathcal{G}}, \phi$ and $\epsilon$ lie below both $\delta$ and $\rho$, the same is true of $\epsilon \circ \phi$, and we are done: we can take $\epsilon \circ \phi$ as the common coatom of $[\varepsilon, \delta]$ and $[\varepsilon, \rho]$ lying over $\epsilon$.

\subsection{Face Enumeration}

Here, we prove formulas counting chains in an oriented interval greedoid $\mathcal{G}$. These results generalize results for oriented matroids [13, Proposition 4.6.2] and for oriented antimatroids [4].

Let $P$ be a poset. Recall that the Möbius function of $P$, denoted $\mu_{P}$, is the unique function from pairs $(x, y)$ with $x \leq y$ in $P$ to $\mathbb{Z}$, such that:

- $\mu_{P}(x, x)=1$.

- For $x<y, \sum_{x \leq z \leq y} \mu_{P}(z, y)=0$.

Theorem 6.8 Let $(E, \mathcal{F}, \mathcal{G})$ be an oriented interval greedoid. Let $A_{1}>\cdots>$ $A_{k+1}=\hat{0}$ be a chain of flats in $\Phi$. Then:

$$
\left|\operatorname{supp}^{-1}\left(A_{1}, \ldots, A_{k+1}\right)\right|=\prod_{i=1}^{k} \sum_{B \in\left[A_{i+1}, A_{i}\right]}\left|\mu_{\Phi}\left(B, A_{i}\right)\right|,
$$

where $\mu_{\Phi}$ is the Möbius function of $\Phi$, and $\operatorname{supp}^{-1}\left(A_{1}, \ldots, A_{k+1}\right)$ denotes the chains $\alpha_{1}>\cdots>\alpha_{k+1}$ in $\mathcal{G}$ such that $\operatorname{supp}\left(\alpha_{i}\right)=A_{i}$.

First, we state and prove the following special case, which generalizes [13, Theorem 4.6.1].

Proposition 6.9 Let $(E, \mathcal{F}, \mathcal{G})$ be an oriented interval greedoid. Then the number of topes of $\mathcal{G}$ is

$$
\sum_{B \in \Phi}\left|\mu_{\Phi}(B, \hat{1})\right|
$$

Proof One could adapt the proof for oriented matroids to this setting, thus reproving the result for oriented matroids, but we prefer to assume the result if $\mathcal{G}$ is an oriented matroid; this is [13, Theorem 4.6.1]. 
Recall that the topes of $\mathcal{G}$ are the same as those of $\overline{\mathcal{G}}$. Applying the proposition to $\overline{\mathcal{G}}$, and writing $\bar{\Phi}$ for $\Phi(\overline{\mathcal{G}})$, we need now only show that

$$
\sum_{B \in \Phi}\left|\mu_{\Phi}(B, \hat{1})\right|=\sum_{B \in \bar{\Phi}}\left|\mu_{\bar{\Phi}}(B, \hat{1})\right| .
$$

Consider the order-preserving map $i: \bar{\Phi} \rightarrow \Phi$ defined by $i([X])=[X]$, as discussed in Sect. 4.2.1. We prove a few more properties of it here.

\section{Lemma 6.10}

(1) $i$ is a poset isomorphism onto its image.

(2) For $A, B \in \bar{\Phi}$, we have $i(A \wedge B)=i(A) \wedge i(B)$.

Proof (1) Proposition 4.7 provides a restriction map from $\Phi$ to $\bar{\Phi}$ defined by $\left.A\right|_{\Gamma(\varnothing)}=\left.\mu\right|_{\Gamma(\varnothing)}(\xi(A) \cap \Gamma(\varnothing))$, which is order-preserving. Since $\left.i(A)\right|_{\Gamma(\varnothing)}=A$, we know $i$ is a poset isomorphism onto its image.

(2) Let $A, B \in \bar{\Phi}$. Let $C=i(A) \wedge i(B)$, and let $D=i\left(\left.C\right|_{\Gamma(\varnothing)}\right)$. It is immediate that $D \geq C$. However, we know $D \leq i(A)$ and $D \leq i(B)$, so $D=C$. This implies that $C$ is in the image of $i$, so, by (1), $C=i(A \wedge B)$.

Thanks to Lemma 6.10 (1), we can identify $\bar{\Phi}$ as a subposet of $\Phi$.

Let $x \in E$ such that $\{x\} \in \mathcal{F}$. Then, by definition, $x \in \Gamma(\varnothing)$. It follows that every coatom of $\Phi$ is in $\bar{\Phi}$. Further, since $\bar{\Phi}$ is a geometric lattice, every element of $\bar{\Phi}$ can be written as a meet (in $\bar{\Phi}$ ) of coatoms. Thanks to Lemma 6.10(2), it follows that $\bar{\Phi}$ consists exactly of those elements of $\Phi$ that can be written as a meet of coatoms in $\Phi$.

\section{Lemma 6.11}

(1) If $A \in \Phi \backslash \bar{\Phi}$, then $\mu_{\Phi}(A, \hat{1})=0$.

(2) If $A \in \bar{\Phi}$, then $\mu_{\Phi}(A, \hat{1})=\mu_{\Phi}(A, \hat{1})$.

Proof (1) Since $A \notin \bar{\Phi}, A$ cannot be expressed as a meet of coatoms of $\Phi$. It follows that the meet of the coatoms of $[A, \hat{1}]$ is strictly greater than $A$. The Crosscut Theorem (see [10]) now implies $\mu_{\Phi}(A, \hat{1})=0$.

(2) We induct on the corank of $A$. The statement is obvious for $A=\hat{1}$. For $A$ of positive corank, we use the formula:

$$
\mu_{\Phi}(A, \hat{1})=-\sum_{A<B \in \Phi} \mu_{\Phi}(B, \hat{1})
$$

Now we observe that, by (1), only the terms with $B \in \bar{\Phi}$ contribute. By induction, these terms agree with $\mu_{\bar{\Phi}}(B, \hat{1})$, which proves the result.

Equation (6.1) is now obvious, and the proposition follows.

Proof of Theorem 6.8 The proof goes exactly as in the oriented matroid case, now that the preparations have been made. $\left|\operatorname{supp}^{-1}\left(A_{k}\right)\right|$ is the number of topes of $\mathcal{G} / A_{k}$, 
which is $\sum_{A_{k} \geq B}\left|\mu\left(B, A_{k}\right)\right|$, and then the rest of the chain lies in $\left.\mathcal{G}\right|_{\xi\left(A_{k}\right)}$, which accounts for the remaining terms.

\section{$6.4 c d$-Index}

Since $\hat{\mathcal{G}}$ is an Eulerian poset, it is natural to ask about its $c d$-index. Using the results of [3], it develops that one can compute the $c d$-index of $\hat{\mathcal{G}}$ directly from the lattice of flats $\Phi$ of $\mathcal{G}$.

We begin by recalling the necessary notions. Let $P$ a graded poset of rank $n+1$, with minimum element $\hat{0}$ and maximum element $\hat{1}$. Let $S$ be a subset of $[n]:=\{1, \ldots, n\}$. The rank-selected subposet $P_{S}$ is the subposet of $P$ consisting only of the elements whose ranks are in $S$. Let $\alpha(S)$ be the total number of maximal chains in $P_{S}$, and let $\beta(S)=\sum_{T \subseteq S}(-1)^{|S|-|T|} \alpha(T)$.

Let $\mathbb{Z}\langle a, b\rangle$ be the noncommutative polynomial ring in the variables $a, b$. Define a monomial $u_{S} \in \mathbb{Z}\langle a, b\rangle$, for $S \subseteq[n]$ by saying that the $i$ th letter in $u_{S}$ is $a$ if $i \notin S$ and it is $b$ if $i \in S$. The $a b$-index of $P$ is the polynomial $\Psi(P)=\sum_{S} \beta(S) u_{S} \in \mathbb{Z}\langle a, b\rangle$.

When $P$ is an Eulerian poset, Fine noticed that $\Psi(P)$ can be expressed as a polynomial with integer coefficients in the noncommuting variables $c=a+b$ and $d=a b+b a$ [12]. The expression in terms of $c$ and $d$ is called the $c d$-index of $P$. If $\Psi(P)$ can be expressed as a polynomial with integer coefficients in $c$ and $2 d$, then the expression is called the $c$ - $2 d$-index of $P$.

There is an operation $\omega: \mathbb{Z}\langle a, b\rangle \rightarrow \mathbb{Z}\langle c, 2 d\rangle$ that acts on monomials by taking each consecutive pair $a b$ and replacing it by $2 d$, and replacing all other letters with $c$. There is also an involution on $\mathbb{Z}\langle a, b\rangle$ that reverses monomials. This involution is denoted by $*$. For $P$ a poset, we denote by $P^{*}$ the dual poset obtained by reversing the relations of $P$. It is clear that $\Psi\left(P^{*}\right)=\Psi(P)^{*}$.

The following follows from Theorem 6.8 and the proof of the main result of [3].

Corollary 6.12 Let $\mathcal{G}$ be an oriented interval greedoid and $\Phi$ its lattice of flats. Then the $c$ - $2 d$-index of $\hat{\mathcal{G}}$ is given by

$$
\Psi(\hat{\mathcal{G}})=\omega\left(a \cdot \Psi\left(\Phi^{*}\right)\right)^{*} .
$$

We close with two final remarks. By Proposition 2.9, $\Phi$ is a lower semimodular lattice. It was shown by Stanley [24] that such posets admit an $R$-labeling, and thus there is a combinatorial interpretation of $\beta(S)[8,25]$.

Our second remark is that the $c$ - $2 d$-index of an Eulerian poset $P$ can also be interpreted as giving the expansion of the Ehrenborg quasi-symmetric function of $P$ [20] with respect to a certain basis, see [6, Corollary 2.2]. From this perspective, the map $\omega$ above corresponds to Stembridge's $\theta$ map [26], which takes quasi-symmetric functions to the peak subalgebra of the quasi-symmetric functions. See [6, Sect. 3.3] for the equivalence between $\omega$ and $\theta$. Corollary 6.12 can therefore be rephrased in the language of quasi-symmetric functions. A version of Corollary 6.12, expressed in those terms, and specialized to the case of oriented antimatroids, is given in [4].

Acknowledgements The authors would like to thank Richard Ehrenborg for pointing out the relevance of [3], and the referee for helpful comments which improved the exposition. H.T. was partially funded by an NSERC Discovery Grant. 


\section{References}

1. Aguiar, M., Mahajan, S.: Coxeter Groups and Hopf Algebras. Fields Institute Monographs, vol. 23. Am. Math. Soc., Providence (2006). With a foreword by Nantel Bergeron. MR2225808 (2008d:20072)

2. Brown, K.S., Diaconis, P.: Random walks and hyperplane arrangements. Ann. Probab. 26(4), 18131854 (1998). MR1675083 (2000k:60138)

3. Billera, L.J., Ehrenborg, R., Readdy, M.: The $c$ - $2 d$-index of oriented matroids. J. Comb. Theory, Ser. A 80(1), 79-105 (1997). MR1472106 (98h:05051)

4. Billera, L.J., Hsiao, S.K., Provan, J.S.: Enumeration in convex geometries and associated polytopal subdivisions of spheres. Discrete Comput. Geom. 39(1-3), 123-137 (2008). MR2383754 (2009b:52046)

5. Bidigare, P., Hanlon, P., Rockmore, D.: A combinatorial description of the spectrum for the Tsetlin library and its generalization to hyperplane arrangements. Duke Math. J. 99(1), 135-174 (1999). MR1700744 (2000m:52032)

6. Billera, L.J., Hsiao, S.K., van Willigenburg, S.: Peak quasisymmetric functions and Eulerian enumeration. Adv. Math. 176(2), 248-276 (2003). MR1982883 (2005a:06003)

7. Bidigare, T.P.: Hyperplane arrangement face algebras and their associated Markov chains. Thesis (Ph.D.) -University of Michigan. ProQuest LLC, Ann Arbor, MI, 1997. MR2695489

8. Björner, A.: Shellable and Cohen-Macaulay partially ordered sets. Trans. Am. Math. Soc. 260(1), 159-183 (1980). MR570784 (81i:06001)

9. Björner, A.: Posets, regular CW complexes and Bruhat order. Eur. J. Comb. 5(1), 7-16 (1984). MR746039 (86e:06002)

10. Björner, A.: Topological methods. In: Handbook of Combinatorics, pp. 1819-1872. Amsterdam, Elsevier (1995). MR1373690 (96m:52012)

11. Björner, A.: Random walks, arrangements, cell complexes, greedoids, and self-organizing libraries. In: Building Bridges. Bolyai Soc. Math. Stud., vol. 19, pp. 165-203. Springer, Berlin (2008). MR2484640 (2010e:05051)

12. Bayer, M.M., Klapper, A.: A new index for polytopes. Discrete Comput. Geom. 6(1), $33-47$ (1991). MR1073071 (91k:52024)

13. Björner, A., Las Vergnas, M., Sturmfels, B., White, N., Ziegler, G.M.: Oriented Matroids. Encyclopedia of Mathematics and Its Applications, vol. 46. Cambridge Univ. Press, Cambridge (1993). MR1226888 (95e:52023)

14. Brown, K.S.: Semigroups, rings, and Markov chains. J. Theor. Probab. 13(3), $871-938$ (2000). MR1785534 (2001e:60141)

15. Brown, K.S.: Semigroup and ring theoretical methods in probability. In: Representations of Finite Dimensional Algebras and Related Topics in Lie Theory and Geometry. Fields Inst. Commun., vol. 40, pp. 3-26. Am. Math. Soc., Providence (2004). MR2057147 (2005b:60118)

16. Björner, A., Wachs, M.: On lexicographically shellable posets. Trans. Am. Math. Soc. 277(1), 323341 (1983). MR690055 (84f:06004)

17. Björner, A., Wachs, M.L.: Shellable nonpure complexes and posets. I. Trans. Am. Math. Soc. 348(4), 1299-1327 (1996). MR1333388 (96i:06008)

18. Björner, A., Ziegler, G.M.: Combinatorial stratification of complex arrangements. J. Am. Math. Soc. 5(1), 105-149 (1992). MR1119198 (92k:52022)

19. Björner, A., Ziegler, G.M.: Introduction to greedoids. In: Matroid Applications. Encyclopedia Math. Appl., vol. 40, pp. 284-357. Cambridge Univ. Press, Cambridge (1992). MR1165545 (94a:05038)

20. Ehrenborg, R.: On posets and Hopf algebras. Adv. Math. 119(1), 1-25 (1996). MR1383883 (97e:16079)

21. Folkman, J., Lawrence, J.: Oriented matroids. J. Comb. Theory, Ser. B 25(2), 199-236 (1978). MR511992 (81g:05045)

22. Korte, B., Lovász, L., Schrader, R.: Greedoids. Algorithms and Combinatorics, vol. 4. Springer, Berlin (1991). MR1183735 (93f:90003)

23. Saliola, F.V.: On the quiver of the descent algebra. J. Algebra 320(11), 3866-3894 (2008). MR2464797 (2009i:20078)

24. Stanley, R.P.: Finite lattices and Jordan-Hölder sets. Algebra Univers. 4, 361-371 (1974). MR0354473 (50 \#6951) 
25. Stanley, R.P.: Enumerative Combinatorics, vol. 1. Cambridge Studies in Advanced Mathematics, vol. 49. Cambridge Univ. Press, Cambridge (1997). With a foreword by Gian-Carlo Rota, Corrected reprint of the 1986 original. MR1442260 (98a:05001)

26. Stembridge, J.R.: Enriched $P$-partitions. Trans. Am. Math. Soc. 349(2), 763-788 (1997). MR1389788 (97f:06006)

27. Steinberg, B.: Möbius functions and semigroup representation theory. J. Comb. Theory, Ser. A 113(5), 866-881 (2006). MR2231092 (2007c:20144) 